\title{
EFFECT OF TIME DELAY IN A CANNIBALISTIC STAGE-STRUCTURED PREDATOR-PREY MODEL WITH HARVESTING OF AN ADULT PREDATOR: THE CASE OF LIONFISH
}

\section{This is a pre print version of the following article:}

Original Citation:

Availability:

This version is available http://hdl.handle.net/2318/1718416

since 2021-12-23T12:44:35Z

Published version:

DOI:10.1142/S0218339019500189

Terms of use:

Open Access

Anyone can freely access the full text of works made available as "Open Access". Works made available under a Creative Commons license can be used according to the terms and conditions of said license. Use of all other works requires consent of the right holder (author or publisher) if not exempted from copyright protection by the applicable law. 


\title{
EFFECT OF TIME DELAY IN A CANNIBALISTIC STAGE-STRUCTURED PREDATOR-PREY MODEL WITH HARVESTING OF AN ADULT PREDATOR: THE CASE OF LIONFISH
}

\author{
BANAMALI MAJI
}

Department of Mathematics, Nayagram Pandit Raghunath Murmu Government College, Nayagram, Baligeria, Jhargram - 721125, India banamalimaji@gmail.com

PANKAJ KUMAR TIWARI*

Department of Mathematics, University of Kalyani, Kalyani - 741235, India pankajmathematics@gmail.com

\section{SUDIP SAMANTA}

Department of Mathematics, Faculty of Science $\&$ Arts - Rabigh, King Abdulaziz University, 13 Rabigh - 25732, Saudi Arabia

samanta.sudip.09@gmail.com

\section{SAMARES PAL}

Department of Mathematics, University of Kalyani, Kalyani - 741235, India samaresp@gmail.com

FRANCESCA BONA

DBIOS, University of Turin, via Accademia Albertina 13, 10123 Turin, Italy francesca.bona@unito.it

The progressive and increasing invasion of an opportunistic predator, the lionfish (Pterois volitans) has become a major threat for the delicate coral reef ecosystem. The herbivore fish populations, in particular of Parrotfish, are taking the consequences of the lionfish invasion and then their control function on macro-algae growth is threatened. In this paper, we developed and analyzed a stage-structured mathematical model including P. volitans (lionfish), a cannibalistic predator, and a Parrotfish, its potential prey. As control upon the over predation, a rational harvest term has been considered. Further, to make the system more realistic a delay in the growth rate of juvenile $P$. volitans population has been incorporated. We performed a global sensitivity analysis to identify important parameters of the system having significant correlations with the fishes. We observed that the system generates transcritical bifurcation which takes the $P$. volitansfree equilibrium to the coexistence equilibrium on increasing the values of predation rate

${ }^{*}$ Corresponding author 


\begin{abstract}
of adult $P$. volitans on Parrotfish. Further increase in the values of the predation rate of adult $P$. volitans on Parrotfish drives the system into Hopf bifurcation, which induces oscillation around the coexistence equilibrium. Moreover, the conversion efficiency due to cannibalism also has the property to alter the stability behavior of the system through Hopf bifurcation. The effect of time delay on the dynamics of the system is extensively studied and it is observed that the system develops chaotic dynamics through perioddoubling oscillations for large values of time delay. However, if the system is already oscillatory, then the large values of time delay causes extinction of $P$. volitans from the system. To illustrate the occurrence of chaotic dynamics in the system, we drew the Poincaré map and also computed the Lyapunov exponents.
\end{abstract}

Keywords: Trophic interactions, Stage-structured population dynamics, Cannibalism, Harvesting, Chaos, Global sensitivity.

\title{
1. Introduction
}

Worldwide almost every organism goes through multiple stages in their life cycle. A stage-structured mathematical model with two stages is identified as juvenile and adult ${ }^{1,2}$. Further, in a stage-structured predator-prey system cannibalistic interactions are very common ${ }^{3,4}$. As the cannibalistic predator invades upon its own species, the predatory pressure on the prey population is reduced ${ }^{5}$. To take shelter by prey is another important phenomenon that has came into the scenario 6,7 . In the coral reef ecosystem, for example, the prey population avoids excessive predation by carnivorous fishes in the seabed by hiding themselves between the coral branches. Based on observations from numerous field and laboratory experiments, ratio-dependent predator-prey models are favored by many researchers $8,9,10,11,12$.

Our model assumes as a predatory species the lionfish (Pterois volitans, Scorpenidae), native to Indo-Pacific. Since 1992 lionfish invaded temperate and tropical of Western Atlantic, starting from Florida through the Bahamas and Caribbean Sea to the Northwestern U.S.A. coasts ${ }^{13,14,15}$. Lionfish are opportunistic and generalist carnivores ${ }^{16}$. Being at the top level of the food chain in numerous reef conditions, $P$. volitans possess a definite life cycle with two stages, juvenile and adult. Lionfish can become mature within the first year of life (when they achieve the total length of 120-200 mm $)^{15}$. Juvenile diet mainly based on shrimps shifts to fish preys when lionfish attempt their sexual maturity ${ }^{16}$. In Western Atlantic, adult diet is mainly based on reef omnivore and carnivore fish some of which are key fishery species ${ }^{14}$. According to Peake et al. ${ }^{16}$, minor is the impact of lionfish on herbivore preys, but on some of them, like red band Parrotfish (Sparisoma aurofrenatum, Scaridae), threatened by overfishing, the lionfish predation could cause a rapid decline. The adult lionfish are known to exhibit a cannibalistic behavior, as they not only feed on the fish preys (mainly Parrotfish), but also on the juveniles of their own species, like many other carnivorous fish. Now-a-days, an acute disruption has shown up in the western Atlantic coral reef ecosystem due to the invasion of lionfish ${ }^{17,18,19}$. Biologically, $P$. volitans show some extraordinary adaptive traits like cryptic form, high competitive nature, fast growth, slow movement, low parasite load etc., which have made them efficient predators and hard to prey on (lionfish are venomous). 
According to Albins ${ }^{20}$, lionfish predation affects native fishes over 2.5 times than that of similar native predator. Moreover, Benkwitt ${ }^{21}$ suggest that lionfish, as their density increases, could show a cooperative hunting behavior, then increasing their efficiency. Lionfish can be an important cause of local or regional decline of herbivores where these latter were stressed by overfishing, like in the Caribbean ${ }^{16}$. As the herbivores feed on algae, loss of herbivores causes a huge spread in algae over the coral reefs, which in turn decelerates the growth of corals ${ }^{20,21,22,23,24}$. Herbivores such as Parrotfish have a key role in the tropical reef ecosystems as they control excessive algae growth thus promoting the biodiversity of the system ${ }^{17,25}$. To control the lionfish invasion in the coral-reef ecosystem, despite the adoption of several techniques of biological control such as introduction of harmful parasites, pathogens and predators, the most effective way to limit their population seems to harvest adults in a particular manner ${ }^{26}$.

A recent study showed that as the herbivorous fish population drops drastically as a consequence of the invasiveness of $P$. volitans, this latter showed a drastic decrease as well ${ }^{21}$. By 2015, in a part of the Bahamas densities of lionfish are declined on most of the reefs, despite of lack of fishing efforts. This oscillatory behaviour of the predator-prey relationship is in accordance with the paradox of biological control in which a system cannot have both a low and stable prey equilibrium density. Bhattacharya and $\mathrm{Pal}^{27}$ investigated the dynamics of a stage-structured predator-prey reaction-diffusion system with Holling type III functional response. They found that the system undergoes Hopf bifurcation when the intrinsic growth rate of herbivorous prey crosses certain critical value. Further, they studied the effects of predation and cannibalism of a top predator species with harvesting ${ }^{28}$. Their analysis leads to different thresholds in terms of the model parameters acting as conditions under which the organisms associated with the system cannot thrive even in the absence of predation. Moreover, they found that the system undergoes Hopf bifurcation when the carrying capacity of macro-algae crosses its critical value.

The evidence of chaos in the real world is still far reaching, but the literature of chaos and chaos control are vast in different fields such as biological systems, electrical engineering, ecological models, and economics $35,36,37,38$. The dynamic relationship between predators and their preys is a dominant theme in both theoretical and mathematical ecology. Without seasonality or structure, chaotic dynamics cannot occur in continuous systems unless at least three species are included. Hastings and Powell ${ }^{38}$ suggested that chaos is common in natural systems (interacting tri-trophic food chain). Occurrence of chaos in a simple ecological system produces chaos as a subject of considerable interest among theoretical ecologists ${ }^{39,40,41}$. However, there is still lack of experimental evidences of chaos in the real world population dynamics. Chaos detection in natural system is difficult due to the presence of observational noise ${ }^{42,43}$. Becks et al. ${ }^{44}$ experimentally showed chaos in the predator-prey interaction between bacterivorous ciliate and two bacterial prey species. Biswas et al. ${ }^{29}$ studied a cannibalistic predator-prey system with a transmissible disease in 
the predator population. They considered incubation delay in disease transmission, and showed that cannibalism can control disease and population oscillations. Further their model analysis reveals that incubation delay destabilizes the system and produces chaos.

Time delay is a very important tool for investigating the real dynamic behavior of a biological system ${ }^{30}$. In reality, the behavior of a system is influenced by both the current and past states of the dynamical variables (i.e., there exists an implicit time lag in the system ${ }^{31,32}$ ). Bhattacharya and Pal ${ }^{33}$ proposed and analyzed a three dimensional stage-structured predator-prey model to study the effect of intraguild predation with harvesting of the adult predators. They considered time lags in reproduction and maturation of the organisms and highlighted that the system undergoes a Hopf-bifurcation when the time lags cross certain critical values. Bhattacharya and Pal ${ }^{34}$ studied a two-dimensional single-species stage-structured model by considering time lags in reproduction (conversion due to cannibalism) and maturation of the organism. They estimated the length of delay preserving the stability of the system and found that stability switches occur for increasing the values of time delays. In this paper, we have modified the model of Bhattacharya and $\mathrm{Pal}{ }^{33}$ by considering the Beddington type functional response for interaction between prey and the adult predator. We have considered only the gestation delay and explored rich dynamics including period doubling bifurcation and chaos, which was absent in earlier work. Numerically we have investigated the combined effects of gestation delay and maturation delay on the stability dynamics and compare our results with previous works.

\section{The mathematical model}

Two fishes, Parrotfish and $P$. volitans are considered for the study of interactions within and among the stage-structured populations. Let $U(t), V(t)$ and $W(t)$ be the abundances of Parrotfish, juvenile $P$. volitans and adult $P$. volitans, respectively, at any time $t>0$. P. volitans are one of the top levels of the food web in many coral reef environments. They are known to feed mostly on small fishes, which include juveniles of their own species. We assume that in the absence of $P$. volitans, the growth of Parrotfish population follows logistic law. Apart from preying on Parrotfish, adult $P$. volitans exhibits a distinct cannibalistic attitude towards its juvenile species; the juvenile $P$. volitans does not attack Parrotfish and has no reproductive ability. We consider that the adult $P$. volitans prey on both Parrotfish and juvenile $P$. volitans following the Beddington type ${ }^{45}$ and Holling type II interactions, respectively. The proliferation of predatory $P$. volitans reduces the population density of herbivorous Parrotfish by changing the community structure of coral reefs for which corals decline with an increase in abundance of seaweeds ${ }^{20}$. Moreover, commercial harvesting of adult $P$. volitans is required to reduce the numbers of $P$. volitans to mitigate their impact on coral reef ecosystems ${ }^{26}$. We consider nonconstant harvesting of the adult $P$. volitans ${ }^{46,47}$. In particular, for a more realistic 
approach, a rational harvesting function has been considered in the model which provides diminishing marginal returns of the harvesting organization. The juvenile and adult $P$. volitans experience natural mortality. Based upon the observations of Castillo-Chavez et al. ${ }^{48}$, it is reasonable to assume that the mortality and maturity rates of fishes are proportional to the number of fishes present in the system. We assume that the reproduction of adult $P$. volitans after uptake of Parrotfish is not instantaneous, but is mediated by some discrete time delay required for egg deposition, embryo development, and hatching ${ }^{49}$.

The schematic diagram for the interplay among all the considered dynamical variables is depicted in Fig. 1. In view of the above ecological assumptions, we have the following system of delay differential equations,

$$
\begin{aligned}
\frac{d U}{d t} & =r U\left(1-\frac{U}{K}\right)-\frac{m_{1} U W}{a_{1} W+b_{1} U+c_{1}}, \\
\frac{d V}{d t} & =\frac{\alpha_{1} m_{1} U(t-\tau) W(t-\tau)}{a_{1} W(t-\tau)+b_{1} U(t-\tau)+c_{1}}-\left(\mu+D_{1}\right) V-\frac{(1-\alpha) m_{2} V W}{a_{2}+V}, \\
\frac{d W}{d t} & =\mu V-D_{2} W-\frac{h W}{c+W} .
\end{aligned}
$$

Here, $a_{2}$ represents the abundance of juvenile $P$. volitans at which reduction in juvenile $P$. volitans is half of the maximum possible reduction that can be ever achieved due to cannibalism. The biological meanings of all the variables and parameters involved in the system (2.1) are given in Table 1. In system (2.1), we have $0<\alpha_{1}, \alpha<1^{50}$. The initial conditions for the system (2.1) take the form

$$
U(\phi)=\psi_{1}(\phi), V(\phi)=\psi_{2}(\phi), W(\phi)=\psi_{3}(\phi),-\tau \leq \phi \leq 0,
$$

where $\psi=\left(\psi_{1}, \psi_{2}, \psi_{3}\right)^{T} \in \mathcal{C}_{+}$such that $\psi_{i}(\phi) \geq 0, i=1,2,3 \forall \phi \in[-\tau, 0]$ and $\mathcal{C}_{+}$denotes the Banach space $\mathcal{C}_{+}\left([-\tau, 0], \mathbf{R}_{+0}^{3}\right)$ of continuous functions mapping the interval $[-\tau, 0]$ into $\mathbf{R}_{+0}^{3}$ and denotes the norm of an element $\psi$ in $\mathcal{C}_{+}$ by $\|\psi\|=\sup _{-\tau \leq \phi \leq 0}\left\{\left|\psi_{1}(\phi)\right|,\left|\psi_{2}(\phi)\right|,\left|\psi_{3}(\phi)\right|\right\}$. For biological feasibility, we further assume that $\psi_{i}(0) \geq 0$ for $i=1,2,3$.

\section{Model without time delay}

In this section, we restrict ourselves to analyze the model in the absence of delay. In the absence of time delay, system (2.1) takes the following form:

$$
\begin{aligned}
\frac{d U}{d t} & =r U\left(1-\frac{U}{K}\right)-\frac{m_{1} U W}{a_{1} W+b_{1} U+c_{1}} \equiv F_{1}(U, V, W), \\
\frac{d V}{d t} & =\frac{\alpha_{1} m_{1} U W}{a_{1} W+b_{1} U+c_{1}}-\left(\mu+D_{1}\right) V-\frac{(1-\alpha) m_{2} V W}{a_{2}+V} \equiv F_{2}(U, V, W), \\
\frac{d W}{d t} & =\mu V-D_{2} W-\frac{h W}{c+W} \equiv F_{3}(U, V, W) .
\end{aligned}
$$




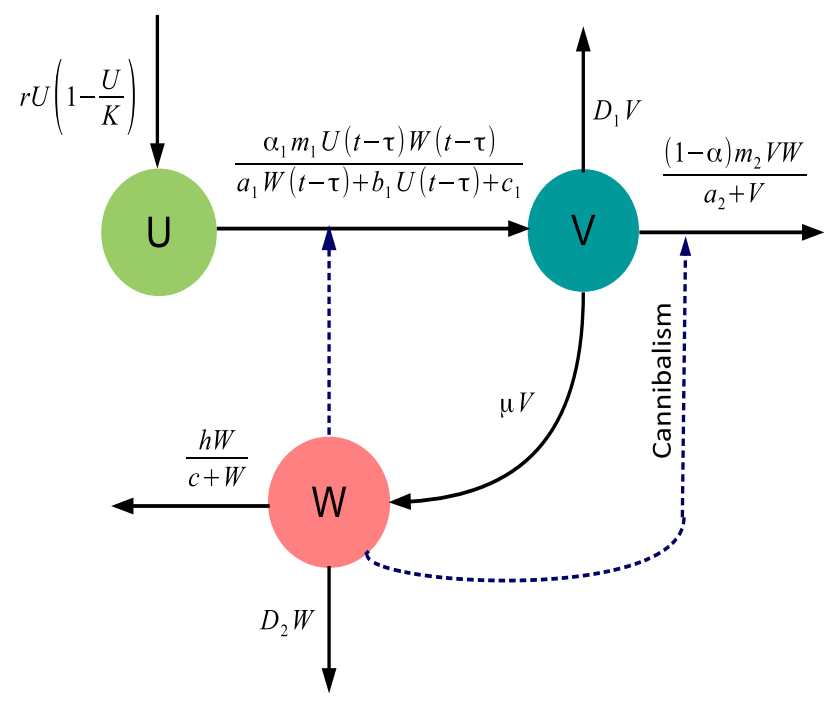

Fig. 1. Schematic diagram of the system (2.1).

Table 1. Biological meanings of variables and parameters in the system (2.1)

\begin{tabular}{|c|c|c|c|}
\hline $\begin{array}{l}\text { Variables/ } \\
\text { Parameters }\end{array}$ & Descriptions & Units & Values \\
\hline$U$ & Abundance of Parrotfish & $\mathrm{mg} / \mathrm{L}$ & - \\
\hline$V$ & Abundance of juvenile $P$. volitans & $\mathrm{mg} / \mathrm{L}$ & - \\
\hline$W$ & Abundance of adult $P$. volitans & $\mathrm{mg} / \mathrm{L}$ & - \\
\hline$r$ & Intrinsic growth rate of Parrotfish population & $1 /$ day & 0.3 \\
\hline$K$ & Carrying capacity of the system & $\mathrm{mg} / \mathrm{L}$ & 10 \\
\hline $1 / \mu$ & Total time spent by $P$. volitans in its juvenile stage & day & 5 \\
\hline$h$ & Maximum harvesting rate of adult $P$. volitans & $\mathrm{mg} / \mathrm{L} /$ day & 0.04 \\
\hline$c$ & Saturation constant for harvesting of adult $P$. volitans & $\mathrm{mg} / \mathrm{L}$ & 0.4 \\
\hline$\alpha_{1}$ & $\begin{array}{c}\text { Growth efficiency of juvenile } P \text {. volitans population due to predation } \\
\text { of Parrotfish by adult } P \text {. volitans }\end{array}$ & - & 0.5 \\
\hline$\alpha$ & $P$. volitans growth efficiency due to cannibalism & - & 0.2 \\
\hline$m_{1}$ & Maximum uptake rate of adult $P$. volitans on Parrotfish & $1 /$ day & 0.5 \\
\hline$m_{2}$ & Maximum uptake rate of adult $P$. volitans on juvenile $P$. volitans & $1 /$ day & 0.21 \\
\hline$a_{1}$ & Scaling the impact of the predator interference & - & 0.1 \\
\hline$b_{1}$ & Food weighting factor & — & 1 \\
\hline$c_{1}$ & $\begin{array}{c}\text { Saturation constant for the uptake of Parrotfish } \\
\text { by adult } P \text {. volitans }\end{array}$ & $\mathrm{mg} / \mathrm{L}$ & 1 \\
\hline$a_{2}$ & $\begin{array}{c}\text { Saturation constant for uptake of juvenile } P \text {. volitans } \\
\text { by adult } P \text {. volitans }\end{array}$ & $\mathrm{mg} / \mathrm{L}$ & 1 \\
\hline$D_{1}$ & Natural death rate of juvenile $P$. volitans & $1 /$ day & 0.005 \\
\hline$D_{2}$ & Natural death rate of adult $P$. volitans & $1 /$ day & 0.1 \\
\hline
\end{tabular}

All parameters involved in the system (3.1) are positive constants. System (3.1) is to be analyzed with the following initial conditions:

$$
U(0)>0, V(0)>0, W(0)>0
$$


Since all the parameters of system (3.1) are non-negative, the right hand side is a smooth function of the variables $U, V$ and $W$ in the positive region

$$
\Theta=\left\{(U, V, W): U, V, W \in \mathbb{R}_{+}\right\} .
$$

\section{Mathematical analysis}

\subsection{Existence, positive invariance, boundedness and persistence of solutions}

Persistence is often a better measure of the type of stability, which is a point of interest of most ecologists, and can be applied under both equilibrium and nonequilibrium conditions. Persistence means that the minimal densities of all species in the focal food web are bounded away from zero, and that they can therefore coexist for a long period of time.

Definition 4.1 System (3.1) is said to be uniformly persistent if there exist finite positive real numbers $M_{1}$ and $M_{2}$ such that each solution of the system (3.1) with positive initial values satisfies

$$
M_{1} \leq \lim _{t \rightarrow \infty} \inf X(t) \leq \lim _{t \rightarrow \infty} \sup X(t) \leq M_{2}, X(t)=(U(t), V(t), W(T)) .
$$

Theorem 4.1 Every solution of system (3.1) with initial conditions (3.2) exists and unique in some interval $[0, \kappa)$, where $U(t)>0, V(t)>0, W(t)>0, \forall t \geq 0$.

The feasible region for system (3.1) is given in the following theorem.

Theorem 4.2 All non-negative solutions of model (3.1) that start in $\mathbb{R}_{+}^{3}$ are uniformly bounded, and the feasible region for system (3.1) is given by the following set

$$
\Omega=\left\{(U, V, W): 0 \leq U+\frac{1}{\alpha_{1}}(V+W) \leq M\right\}
$$

which is compact and invariant with respect to system (3.1).

The following theorem rules out the possibility of extinction of any organism in the system under suitable conditions.

Theorem 4.3 For large $t$, if $r>\frac{m_{1}}{a_{1}}$, then there exist

$u_{1}>K\left(1-\frac{m_{1}}{r a_{1}}\right), v_{1}>\frac{1}{\mu}\left(D_{2} M_{2}+h\right), w_{1}>\frac{M_{1}\left(\mu+D_{1}\right)\left(a_{1} M_{2}+b_{1} K+c_{1}\right)}{\alpha_{1} m_{1} u_{1}-m_{2}(1-\alpha)\left(a_{1} M_{2}+b_{1} K+c_{1}\right)}$

such that

$$
u_{1} \leq U(t) \leq K, v_{1} \leq V(t) \leq M_{1}, w_{1} \leq W(t) \leq M_{2}
$$

For proofs of Theorems 4.1, 4.2 and 4.3, see Appendix A. 


\subsection{System's equilibria}

Due to nonlinearity of system (3.1), it is not possible to find exact solutions to the system. Instead, we settle for determining the long-term behavior of the system. In general, a nonlinear system either gravitates towards an equilibrium point or it blows up. The equilibrium points are those states of dynamical system at which system does not move. Once the system reaches at an equilibrium state, it freeze at this state for all future times. These points can be obtained by putting the growth rate of different variables of model system equal to zero.

System (3.1) has the following three non-negative equilibria:

(1) Organism-free equilibrium $E_{0}=(0,0,0)$, which is always feasible.

(2) P. volitans-free equilibrium $E_{1}=(K, 0,0)$, which is always feasible.

(3) Coexistence equilibrium $E^{*}=\left(U^{*}, V^{*}, W^{*}\right)$, where $U^{*}$ is a positive root of the following algebraic equation:

$$
\frac{\alpha_{1} m_{1} U \theta(U)}{a_{1} \theta(U)+b_{1} U+c_{1}}-\left(\mu+D_{1}\right) \phi(U)-\frac{(1-\alpha) m_{2} \phi(U) \theta(U)}{a_{2}+\phi(U)}=0,
$$

with

$$
\theta(U)=\frac{r(K-U)\left(b_{1} U+c_{1}\right)}{m_{1} K-r a_{1}(K-U)}, \phi(U)=\frac{\theta(U)}{\mu}\left(D_{2}+\frac{h}{c+\theta(U)}\right) ; W^{*}=\theta\left(U^{*}\right), V^{*}=\phi\left(U^{*}\right) .
$$

Remark 4.1 In the equilibrium $E_{0}$, where there is no species in the system, it should not be possible to observe it i.e., this equilibrium point should be unstable. In the equilibrium $E_{1}$, where there is no P. volitans in the system, it should be possible to observe it in some special circumstances i.e., this equilibrium can be stable under certain conditions. Finally, the equilibrium $E^{*}$, where all species are present, is very common in nature, thus it should be stable in some ecosystems.

\subsection{Local stability of equilibria}

In this section, we perform the local stability analysis of the equilibria of system (3.1). This analysis provides excellent information about the behavior of a dynamical system. The local stability analysis characterizes whether or not the system settles to the equilibrium point if its state is initiated close to, but not precisely at a given equilibrium point. The equilibrium point is said to be locally asymptotically stable if there is a neighborhood of the equilibrium point such that for all initial starts in this neighborhood, the system approaches to the equilibrium point as $t \rightarrow \infty$. The local stability of an equilibrium can be investigated by determining the sign of the eigenvalues of Jacobian matrix evaluated at the equilibrium.

Regarding local stability of equilibria of the system (3.1), we have the following theorem.

Theorem 4.4 (1) The equilibrium $E_{0}=(0,0,0)$ is always unstable. Thus, under no circumstances the system (3.1) collapses. 
(2) The equilibrium $E_{1}=(K, 0,0)$ is stable if $m_{1}<m_{1}^{*}$ and unstable if $m_{1}>m_{1}^{*}$, where $m_{1}^{*}=\frac{\left(b_{1} K+c_{1}\right)\left(\mu+D_{1}\right)}{\mu \alpha_{1} K}\left(D_{2}+\frac{h}{c}\right)$. Thus, with low uptake rate of adult P. volitans on Parrotfish (or with high harvesting rate of adult P. volitans), the system stabilizes at the equilibrium $E_{1}$.

(3) The equilibrium $E^{*}=\left(U^{*}, V^{*}, W^{*}\right)$, if feasible, is locally asymptotically stable provided the following conditions are satisfied:

$$
A_{1}>0, A_{2}>0, A_{1} A_{2}-A_{3}>0,
$$

where $A_{i}$ 's are defined in the proof.

For proof of this theorem, see Appendix B.

Remark 4.2 The last point of Theorem 4.4 tells that if the initial state of system (3.1) is near the equilibrium point $E^{*}$, then the solution trajectories not only stay near $E^{*}$ for all $t>0$ but also approaches to $E^{*}$ as $t \rightarrow \infty$. Thus, if the initial value of state variables $U, V$ and $W$ are close to $U^{*}, V^{*}$ and $W^{*}$, respectively, then the system (3.1) will eventually get stabilized provided condition (4.2) holds.

We proved in Theorem 4.4 that if $m_{1}<m_{1}^{*}$, the $P$. volitans-free equilibrium $E_{1}$ is locally asymptotically stable. When the condition is reversed, then the equilibrium $E_{1}$ losses stability and one can prove the feasibility of a coexistence equilibrium.

Theorem 4.5 System (3.1) has a coexistence equilibrium if $m_{1}>m_{1}^{*}$.

Proof. We observed from Theorem 4.2 that the system (3.1) is dissipative. On the other hand, whenever $m_{1}>m_{1}^{*}$, Theorem 4.4 implies that the $P$. volitans-free equilibrium $E_{1}$ is unstable, and therefore the solutions move away from the equilibrium $E_{1}$, which is equivalent to uniform persistence of the system (3.1) (see Theorem 4.3 in Freedman et al. ${ }^{51}$, where the equilibrium $E_{1}$ plays the role of the invariant set $N$; and invariant set $N$ refers to the maximal invariant set on the boundary). Consequently, according to Theorem D.3 in Smith and Paul ${ }^{52}$, the invariance of $\Omega$ and the uniform persistence guarantee the feasibility of the coexistence equilibrium, $E^{*}$. One can easily check that $\left\{E_{1}\right\}$ is the maximal invariant set on $\partial \Omega$.

\subsection{Transcritical bifurcation}

In the context of a biological system, bifurcations describe how the system dynamics may qualitatively drastically change if a parameter varies. A transcritical bifurcation is a local bifurcation, in which a fixed point exists for all values of a parameter and is never destroyed. However, such a fixed point interchanges its stability with another fixed point as the parameter is varied. In other words, both before and after the bifurcation, there is one unstable and one stable fixed point. However, their stability is exchanged when they collide; so, the unstable fixed point becomes stable and vice versa. 
In the previous section, we proved that when $m_{1}<m_{1}^{*}$, then the $P$. volitans-free equilibrium $E_{1}$ is a stable equilibrium of system (3.1). If the condition is reversed, then the $P$. volitans-free equilibrium losses its stability and the coexistence equilibrium $E^{*}$ emerges. That is, if we consider $m_{1}$ as a bifurcation parameter, then at $m_{1}=m_{1}^{*}$, it is to be expected an exchange of stability properties between these two equilibria. This is a clear indication of the presence of a transcritical bifurcation when $m_{1}=m_{1}^{*}$. In the following, we prove that indeed the system (3.1) undergoes a transcritical bifurcation around the equilibrium $E_{1}$ at $m_{1}=m_{1}^{*}$.

As observed previously, the equilibrium $E_{1}$ is locally stable if $m_{1}<m_{1}^{*}$ and unstable if $m_{1}>m_{1}^{*}$. As a consequence, the critical value $m_{1}=m_{1}^{*}$ is a bifurcation value. The next step is to investigate the nature of the bifurcation involving $E_{1}$ at $m_{1}=m_{1}^{*}$. In view of previous considerations, we have the following theorem.

Theorem 4.6 Consider system (3.1) and let $a$ and $b$ as given by (6.4), where $b>0$. The local dynamics of system (3.1) around the equilibrium $E_{1}$ are totally determined by the sign of $a$.

(1) If $a<0$, when $m_{1}<m_{1}^{*}$ with $m_{1} \approx m_{1}^{*}$, the equilibrium $E_{1}$ is locally asymptotically stable, and there exists a negative unstable equilibrium $E^{*}$; when $m_{1}>m_{1}^{*}$ with $m_{1} \approx m_{1}^{*}$, the equilibrium $E_{1}$ is unstable, and there exists a positive locally asymptotically stable equilibrium $E^{*}$.

(2) If $a>0$, when $m_{1}<m_{1}^{*}$ with $m_{1} \approx m_{1}^{*}$, the equilibrium $E_{1}$ is locally asymptotically stable, and there exists a positive unstable equilibrium $E^{*}$; when $m_{1}>m_{1}^{*}$ with $m_{1} \approx m_{1}^{*}$, the equilibrium $E_{1}$ is unstable, and there exists a negative locally asymptotically stable equilibrium $E^{*}$.

Proof. It follows from Castillo-Chavez and Song ${ }^{53}$ Theorem 4.1 pp. 373, and Remark 1 pp. 375.

Corollary 4.1 Consider system (3.1) and let $a$ and $b$ as given by (6.4) where $b>0$. At $m_{1}=m_{1}^{*}$, system (3.1) undergoes a transcritical bifurcation. If $a<0$, the bifurcation at $m_{1}=m_{1}^{*}$ is supercritical (or forward). On the other hand, if $a>0$, the bifurcation at $m_{1}=m_{1}^{*}$ is subcritical (or backward) bifurcation.

Proof. It is a straightforward application of Theorem 4.6.

The expressions for quantities $a$ and $b$ used in Theorem 4.6 are derived in $A p$ pendix $C$.

\subsection{Nonexistence of periodic solutions and global stability}

It is important to observe whether there is any periodic solution of an ecological system as the existence of periodic solution can derive complex ecological phenomena. On the other hand, nonexistence of periodic solution can enable to make a 
locally stable equilibrium into a globally stable equilibrium. In this section, we extend our stability analysis beyond the small region near equilibrium point to the whole region of attraction using Lyapunov's second method. The basic idea of this technique for verifying nonlinear stability of equilibrium point is to seek an energy function that decreases with time along the trajectories of the system.

The following theorem states the condition for the nonexistence of periodic solutions of the system (3.1) around the coexistence equilibrium $E^{*}$.

Theorem 4.7 The system (3.1) has no periodic solution around the equilibrium $E^{*}$ if

$$
\begin{gathered}
\mu+\frac{m_{1} U^{*}\left[2 b_{1} W^{*}+\left(\alpha_{1}+1\right)\left(b_{1} U^{*}+c_{1}\right)\right]+\alpha_{1} m_{1} W^{*}\left(a_{1} W^{*}+c_{1}\right)}{\left(a_{1} W^{*}+b_{1} U^{*}+c_{1}\right)^{2}}< \\
\min \left\{\mu+D_{1}+\frac{r U^{*}}{K}+\frac{m_{2} a_{2}(1-\alpha) W^{*}}{\left(a_{2}+V^{*}\right)^{2}}+\frac{m_{2}(1-\alpha) V^{*}}{a_{2}+V^{*}}, D_{2}+\frac{r U^{*}}{K}+\frac{h c}{\left(c+W^{*}\right)^{2}},\right. \\
\left.\mu+D_{1}+D_{2}+\frac{h c}{\left(c+W^{*}\right)^{2}}+\frac{m_{2} a_{2}(1-\alpha) W^{*}}{\left(a_{2}+V^{*}\right)^{2}}\right\} .
\end{gathered}
$$

Regarding global stability of the equilibrium $E^{*}$, we have the following theorem.

Theorem 4.8 The coexistence equilibrium $E^{*}$, if feasible, is globally asymptotically stable inside the region of attraction $\Omega$ if the following conditions hold,

$$
\begin{aligned}
& {\left[\frac{\alpha_{1}^{2} m_{1} M\left(a_{1} W^{*}+c_{1}\right)}{c_{1}\left(a_{1} W^{*}+b_{1} U^{*}+c_{1}\right)}\right]^{2}<\left(\mu+D_{1}\right)\left[\frac{r}{K}-\frac{m_{1} b_{1} \alpha_{1} M}{c_{1}\left(a_{1} W^{*}+b_{1} U^{*}+c_{1}\right)}\right],} \\
& {\left[\frac{m_{1}\left(b_{1} M+c_{1}\right)}{c_{1}\left(a_{1} W^{*}+b_{1} U^{*}+c_{1}\right)}\right]^{2}<\left[\frac{r}{K}-\frac{m_{1} b_{1} \alpha_{1} M}{c_{1}\left(a_{1} W^{*}+b_{1} U^{*}+c_{1}\right)}\right]\left[D_{2}+\frac{h c}{\left(c+\alpha_{1} M\right)\left(c+W^{*}\right)}\right],} \\
& {\left[\mu+\frac{\alpha_{1} m_{1} U^{*}\left(b_{1} M+c_{1}\right)}{c_{1}\left(a_{1} W^{*}+b_{1} U^{*}+c_{1}\right)}-\frac{m_{2}(1-\alpha) V^{*}}{a_{2}+V^{*}}\right]^{2}<\left(\mu+D_{1}\right)\left[D_{2}+\frac{h c}{\left(c+\alpha_{1} M\right)\left(c+W^{*}\right)}\right] .}
\end{aligned}
$$

Remark 4.3 If conditions in (4.4) are satisfied, then it guarantees that for every initial start within the region of attraction $\Omega$, solution trajectories will reach to the equilibrium state $E^{*}$ (i.e. the abundances of Parrotfish and P. volitans will get stabilized). Note that for the set of parameter values given in Table 1 except $c=0.05$, the conditions in (4.3) are satisfied, which guarantee that there exists no periodic solution of the system (3.1) for this set of parameter values.

For proofs of Theorems 4.7 and 4.8, see Appendix D.

\subsection{Existence of Hopf bifurcation}

In this section, we investigate for the possibility of Hopf bifurcation from the coexistence equilibrium $E^{*}$ by taking the uptake rate of $P$. volitans on Parrotfish $\left(m_{1}\right)$ as bifurcation parameter and keeping other parameters fixed. A Hopf bifurcation is a critical point where a system's stability switches and a periodic solution arises.

We have the following result regarding the existence of Hopf-bifurcation. 
Theorem 4.9 The necessary and sufficient conditions for the occurrence of Hopf bifurcation from the coexistence equilibrium $E^{*}$ is that there exists $m_{1}=m_{1}^{c}$ such that

$$
\begin{aligned}
& \text { (i) } H\left(m_{1}^{c}\right)=A_{1}\left(m_{1}^{c}\right) A_{2}\left(m_{1}^{c}\right)-A_{3}\left(m_{1}^{c}\right)=0, \\
& \text { (ii) }\left[\frac{d}{d m_{1}}\left(A_{1} A_{2}-A_{3}\right)\right]_{m_{1}=m_{1}^{c}} \neq 0 .
\end{aligned}
$$

For proof of this theorem, see Appendix E.

\subsection{Stability analysis in the presence of time delay}

In this section, we study the local stability of the delayed system (2.1) around the coexistence equilibrium point only. The following theorem gives a criterion for the switching in the stability behavior of equilibrium $E^{*}$ in terms of the delay parameter $(\tau)$.

Theorem 4.10 Suppose that the coexistence equilibrium point $E^{*}$ is feasible and locally asymptotically stable for $\tau=0$. Also, let $\theta_{0}=\omega_{0}^{2}$ be a positive root of (6.18), then there exists $\tau=\tau^{*}$ such that the coexistence equilibrium point $E^{*}$ of the delay system (2.1) is asymptotically stable when $0 \leq \tau<\tau^{*}$ and unstable for $\tau>\tau^{*}$. Furthermore, the system will undergo a Hopf bifurcation at the equilibrium $E^{*}$ when $\tau=\tau^{*}$, provided $U(\omega) R(\omega)-S(\omega) W(\omega)>0$.

For proof of this theorem, see Appendix F.

\section{Numerical simulation}

Here, we report the simulations to investigate the behavior of systems (2.1) and (3.1), performed using MatLab. The set of parameter values are chosen within the range prescribed in various previous literature sources ${ }^{24,27,28,33,34}$, and are given in Table 1. Values of parameters are arbitrary and have been used for illustration purpose only. Unless it is mentioned, the values of parameters used for numerical simulations are the same as in Table 1.

\subsection{Sensitivity analysis}

To identify the most influential parameters that have significant impact on output variables of the system (3.1), we perform global sensitivity analysis ${ }^{54,55}$. We calculate partial rank correlation coefficients (PRCCs) between the parameters $m_{1}$, $a_{1}, \alpha_{1}, \alpha, m_{2}, a_{2}, h$ and $c$ from system (3.1) with Parrotfish, juvenile P. volitans and adult $P$. volitans. Nonlinear and monotone relationships are observed with the input parameters of the model (3.1), which is a prerequisite for computing PRCCs. Then a total of 200 simulations of the model per LHS run were carried out, using 
Table 2. Ranges of variability of the considered sensitive parameters of the system (3.1)

\begin{tabular}{cccc}
\hline Parameters & Baseline values & Minimum values & Maximum values \\
\hline$m_{1}$ & 0.52 & 0.39 & 0.65 \\
$a_{1}$ & 0.1 & 0.075 & 0.125 \\
$\alpha_{1}$ & 0.5 & 0.375 & 0.625 \\
$\alpha$ & 0.2 & 0.15 & 0.25 \\
$m_{2}$ & 0.21 & 0.1575 & 0.2625 \\
$a_{2}$ & 1 & 0.75 & 1.25 \\
$h$ & 0.04 & 0.3 & 0.05 \\
$c$ & 0.4 & 0.3 & 0.5 \\
\hline
\end{tabular}
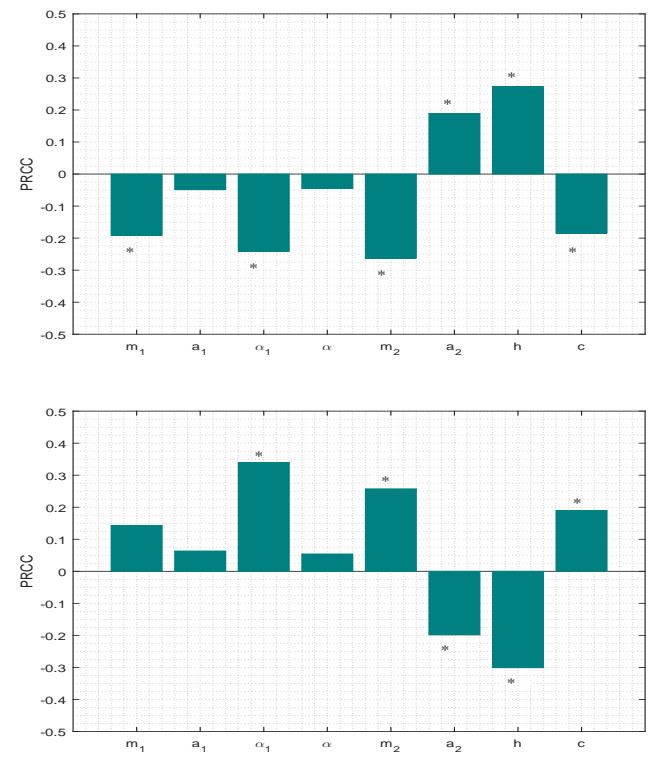

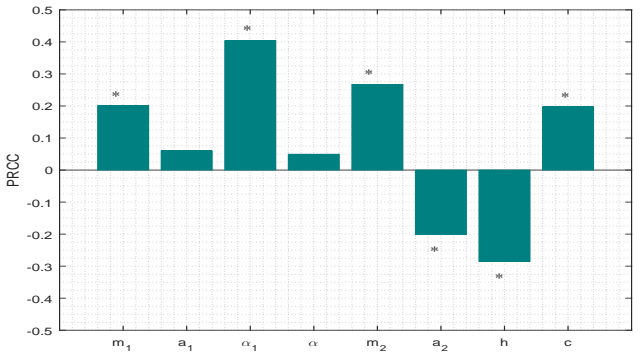

a

$\mathrm{b}$

Fig. 2. Effect of uncertainty of the model (3.1) on (a) Parrotfish, (b) juvenile P. volitans and (c) adult $P$. volitans. Each bar represents the uncertainty of the populations with respect to particular parameters for the model (3.1). 200 samples for each parameter were drawn using Latin Hypercube Sampling technique; parameter ranges are given in Table 2. Significant parameters are marked by

the baseline values tabulated in Table 2 and the ranges as $25 \%$ from the baseline values (in either direction). Note that the PRCC values lie between -1 and 1 . Positive (negative) values indicate a positive (negative) correlation of the parameter with the model output. A positive (negative) correlation implies that a positive (negative) change in the parameter will increase (decrease) the model output. The larger the absolute value of the PRCC, the greater the correlation of the parameter 
with the output. The bar diagram of the PRCC values of Parrotfish, juvenile $P$. volitans and adult $P$. volitans against the parameters is depicted in Fig. 2. PRCC values of parameters with the responses suggest that the parameters $m_{1}, \alpha_{1}, m_{2}, a_{2}$, $h$ and $c$ have significant correlations with Parrotfish and juvenile $P$. volitans. The parameters $\alpha_{1}, m_{2}, a_{2}, h$ and $c$ have significant correlations with adult $P$. volitans. The parameters $a_{2}$ and $h$ have positive correlations with the Parrotfish while other parameters have negative correlations. In contrast, $a_{2}$ and $h$ have negative correlations with juvenile and adult $P$. volitans while other parameters have positive correlations with these populations.

\subsection{Dynamics of system in the absence of time delay}
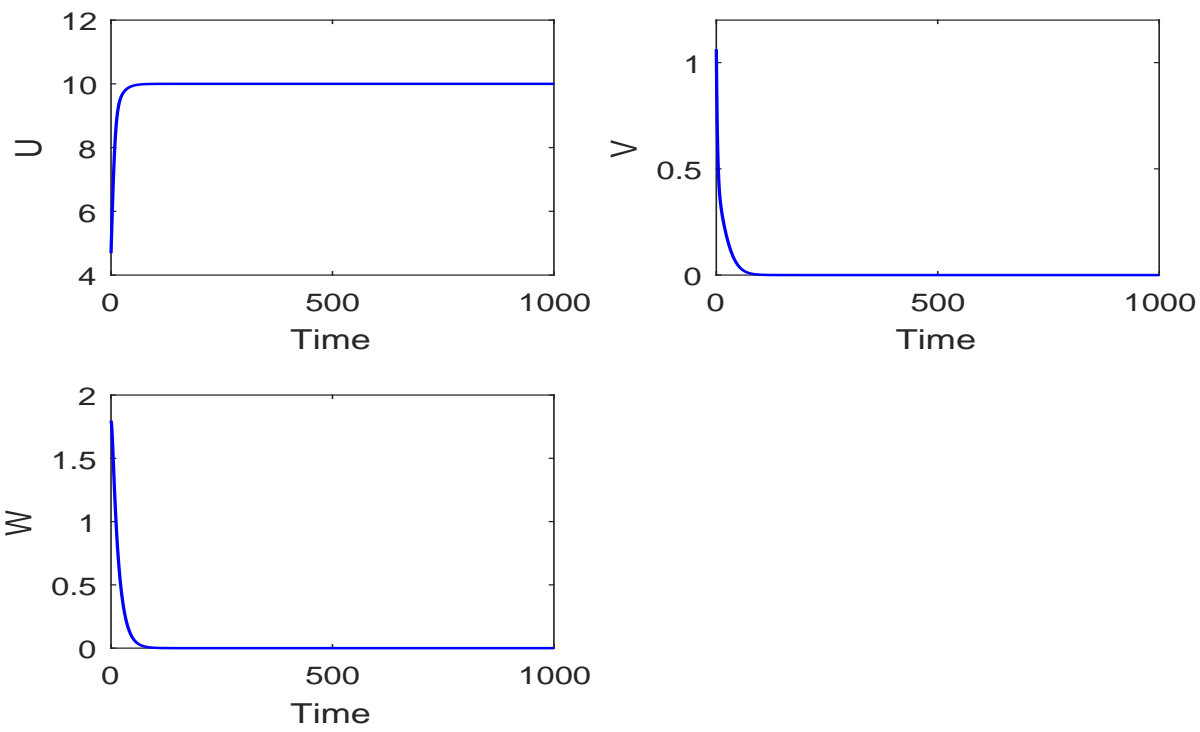

Fig. 3. Equilibrium $E_{1}$ is achieved at $m_{1}=0.2$. Rest of the parameter values are same as in Table 1 .

In this section, we see how different dynamics occur by varying two parameters of the system (3.1): predation rate of adult $P$. volitans on Parrotfish $\left(m_{1}\right)$ and the conversion efficiency due to cannibalism $(\alpha)$, fixing the values of rest of the parameters as in Table 1. We observe that for the set of parameter values given in Table 1 , system (3.1) possesses unique positive equilibrium $E^{*}=(5.7258,1.0482,1.7703)$. At $m_{1}=0.2$, we get that the equilibrium $E_{1}=(10,0,0)$ is stable (see Fig. 3$)$ but losses its stability with increase in the values of $m_{1}$ and the equilibrium $E^{*}$ becomes feasible. With high invasiveness of adult $P$. volitans, the maximal uptake rate of 

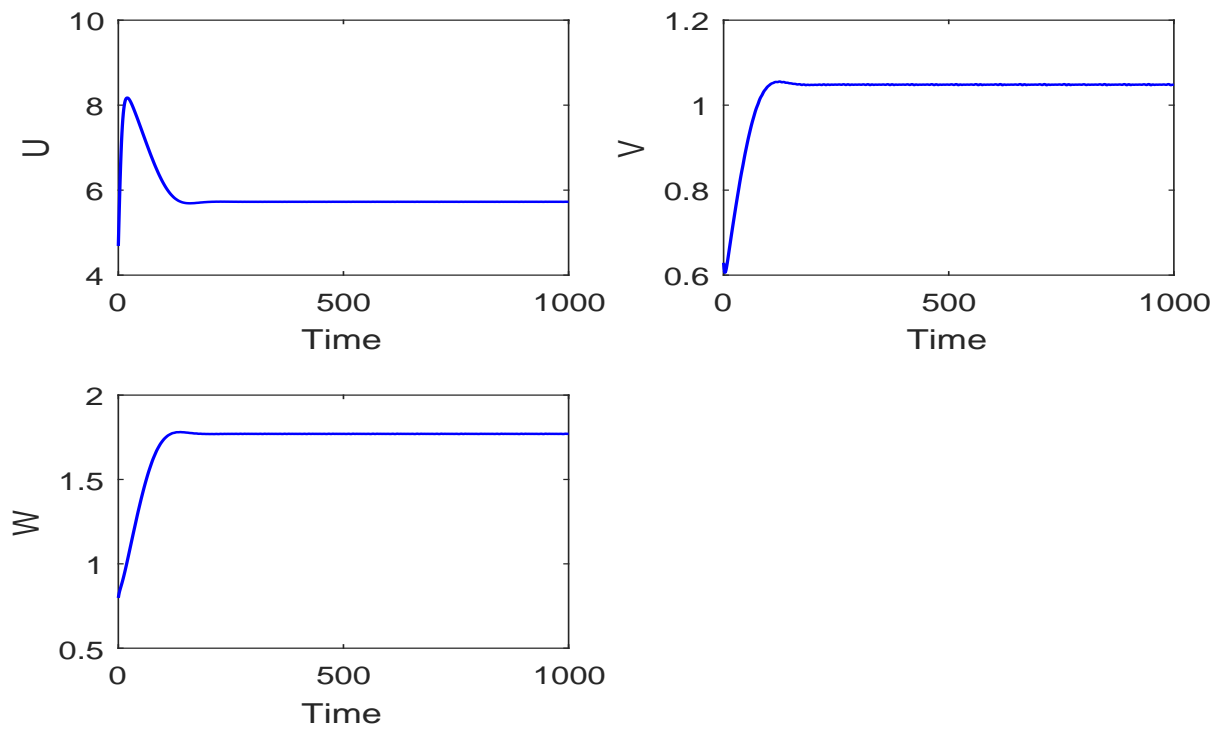

Fig. 4. Equilibrium $E^{*}$ is stable at $m_{1}=0.5$. Rest of the parameter values are same as in Table 1.

adult $P$. volitans on Parrotfish becomes high. At $m_{1}=0.5$, the equilibrium $E^{*}$ is stable focus (see Fig. 4) but when we increase the values of $m_{1}$ to $m_{1}=0.54$, the system (3.1) develops limit cycle oscillations, Fig. 5. This confirms that the system (3.1) undergoes Hopf bifurcation through equilibrium $E^{*}$. The occurrence of Hopf bifurcation might be a warning that the ecosystem could become endangered, if the amplitude of these oscillations grows. Indeed, if the troughs become too low and are thus close to zero, environmental stochastic perturbations may push the oscillating population to extinction, in spite of the fact that mathematically it should continue to oscillate. Next, we keep $m_{1}$ fixed at $m_{1}=0.5$ (where the equilibrium $E^{*}$ is stable focus) and increase the values of $\alpha$. We find that at $\alpha=0.35$, the system (3.1) shows limit cycle oscillations around the equilibrium $E^{*}$, Fig. 6. Thus, the parameters $m_{1}$ and $\alpha$ can change the stability behaviors of the system (3.1). To be more clear about the roles of $m_{1}$ and $\alpha$ on the change of stability, we draw bifurcation diagrams of the system (3.1) with respect to $m_{1}$ and $\alpha$, Figs. 7 and 8 , respectively. From Fig. 7 , it is evident that for low values of $m_{1}$, the equilibrium $E_{1}$ is stable but losses its stability and the equilibrium $E^{*}$ becomes stable with increase in the values of $m_{1}$. Further increase in the values of $m_{1}$ makes the equilibrium $E^{*}$ unstable. The critical value of $m_{1}$ at which transcritical and Hopf bifurcations occur are $m_{1}^{*} \approx 0.426$ and $m_{1}^{c} \approx 0.528$, respectively. Next, we fix $m_{1}=0.5$ and gradually increase the values of $\alpha$. We find that for low values of $\alpha$, the equilibrium $E^{*}$ is stable but losses its stability after a threshold value of $\alpha$. The critical value of $\alpha$ at which stability changes is $\alpha^{c} \approx 0.305$. Previously existence of Hopf bifurcation is 

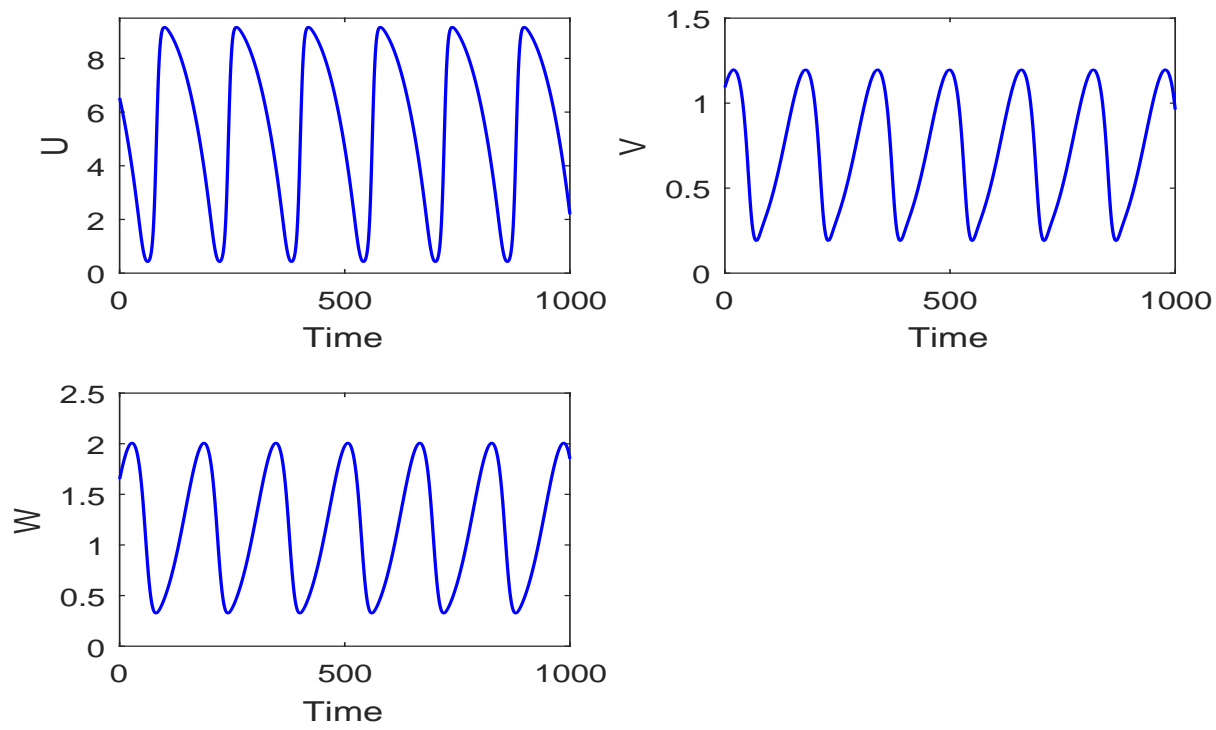

Fig. 5. Equilibrium $E^{*}$ is unstable at $m_{1}=0.54$. Rest of the parameter values are same as in Table 1.

shown by taking the intrinsic growth rate ${ }^{27}$ and carrying capacity ${ }^{28}$ of Parrotfish as bifurcation parameters. In these studies, Holling type III and ratio-dependent interactions were considered.

\subsection{Dynamics of the system in the presence of time delay}

To see the effect of time delay involved in the growth of juvenile $P$. volitans, we set the system (2.1) at stable focus in the absence of time delay. We gradually increase the values of time delay $(\tau)$ and plotted the solution trajectories of the system (2.1). We observe that the system (2.1) is stable focus at $\tau=1$, Fig. 9 . Now, we increase the values of time delay to $\tau=60$ and find that the system (2.1) exhibits limit cycle oscillations, Fig. 10. Next, we increase the values of time delay to $\tau=125$ and get that the system exhibits period doubling oscillations, Fig. 11 . The system shows chaotic behavior for further increase in time delay $(\tau=139)$, Fig. 12. For better visualization, we draw the bifurcation diagram of the system (2.1) by varying the bifurcation parameter $\tau$ in the interval [1,139], Fig. 13. It is clear from the figure that the system shows stable focus for $\tau<50$, limit cycle behaviour for $50 \leq \tau \leq 121$, period doubling oscillations for $121 \leq \tau \leq 128$, and higher periodic and chaotic oscillations for $\tau \geq 128$. The chaotic regime is reached via stable focus to limit cycle oscillation, period doubling oscillations and eventually leads to chaos. Further, we draw the Poincaré map of the system (2.1) on $V-W$ plane $(U=4.5)$ for $\tau=139$, Fig. 14. The scattered distribution of the sampling 

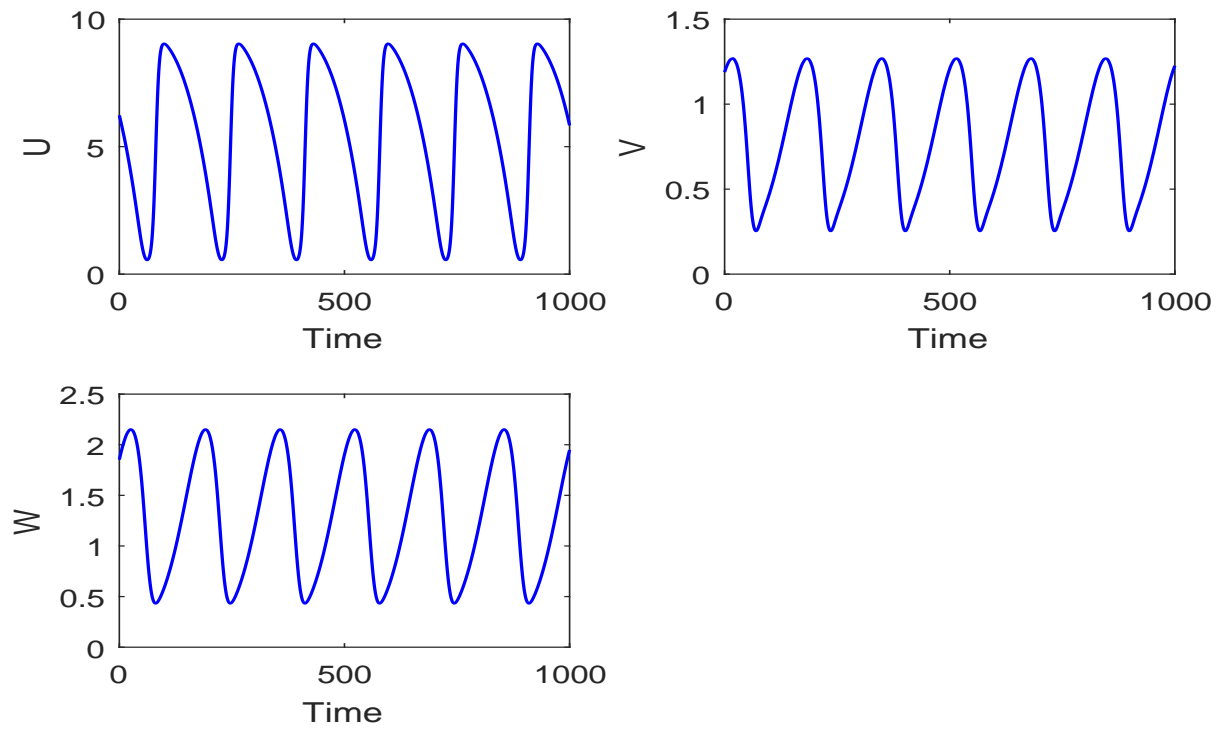

Fig. 6. System (3.1) shows limit cycle oscillation around the equilibrium $E^{*}$ at $\alpha=0.35$. Rest of the parameter values are same as in Table 1 .

points implies the chaotic behavior of the system. We also draw the maximum Lyapunov exponent of the system (2.1) for $\tau=139$, Fig. 15. To draw the maximum Lyapunov exponent, we first simulate the delayed system (2.1), then considering the time series solutions of each component, we compute the Lyapunov exponents 56,57 . In the figure, positive values of the maximum Lyapunov exponent indicates the chaotic regime of the system. Therefore, we can conclude that the system (2.1) shows chaotic behavior for $\tau=139$.

Next, we see the effect of time delay in an oscillating system. We fixed the values of $m_{1}$ at $m_{1}=0.53$ and vary the values of time delay, Fig. 16 . We observe that at $\tau=0$, the system shows oscillatory behavior; at $\tau=10$, oscillations are still there but the period and amplitude of oscillations increase; at $\tau=50$, the $P$. volitans populations extinct.

Earlier, Bhattacharya and $\mathrm{Pal}{ }^{33}$ investigated a similar stage-structured model with gestation delay and maturation delay, and constant harvesting. They observed that delay can destabilize the system by producing limit cycle oscillations, whereas harvesting can stabilize the system. In the present work, for a different set of parameter values, we explored rich dynamics including chaos for gradual increase of gestation delay. Analytically we have studied the stage-structured model with gestation delay only while harvesting follows a saturating functional response. To explore the impact of maturation delay, we extend the model with maturation delay and 

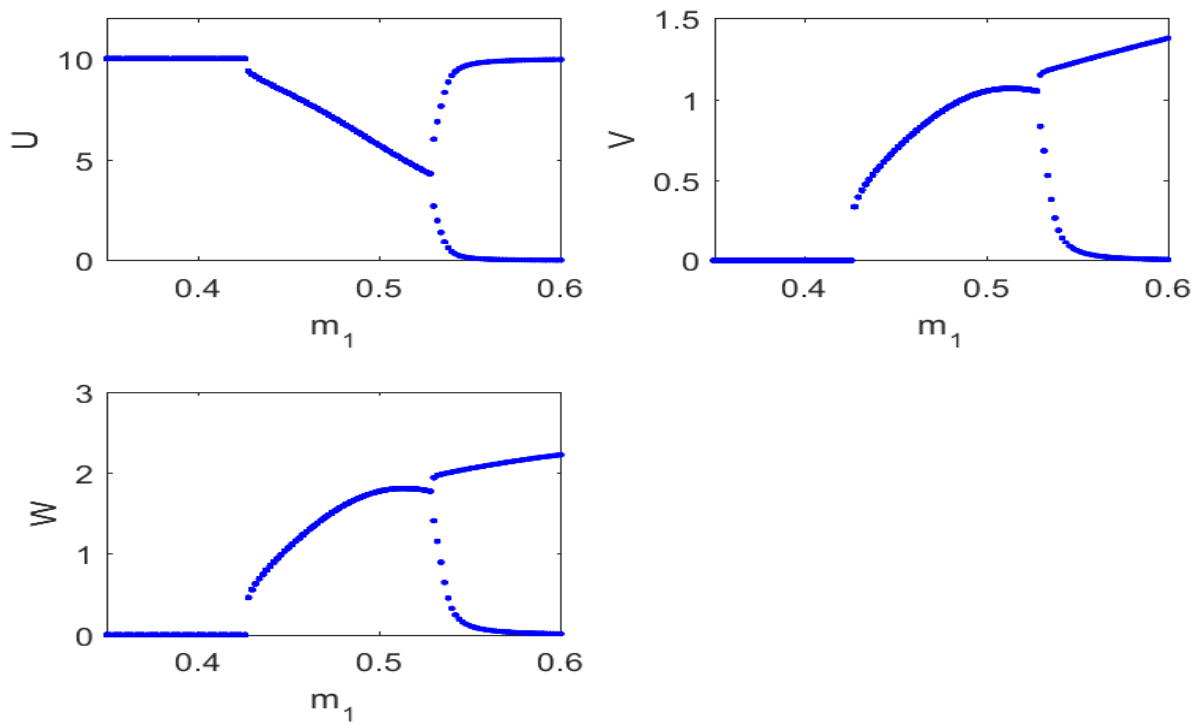

Fig. 7. Bifurcation diagram of the system (3.1) with respect to $m_{1}$. Rest of the parameter values are same as in Table 1.

perform numerical simulations. The system with two delays becomes,

$$
\begin{aligned}
\frac{d U}{d t} & =r U\left(1-\frac{U}{K}\right)-\frac{m_{1} U W}{a_{1} W+b_{1} U+c_{1}}, \\
\frac{d V}{d t} & =\frac{\alpha_{1} m_{1} U\left(t-\tau_{1}\right) W\left(t-\tau_{1}\right)}{a_{1} W\left(t-\tau_{1}\right)+b_{1} U\left(t-\tau_{1}\right)+c_{1}}-\left(\mu+D_{1}\right) V-\frac{(1-\alpha) m_{2} V W}{a_{2}+V}, \\
\frac{d W}{d t} & =\mu V\left(t-\tau_{2}\right)-D_{2} W-\frac{h W}{c+W} .
\end{aligned}
$$

Note that here $\tau_{1}$ is $\tau$ in system (2.1). The dynamics of system (5.1) is depicted in Fig. 17. We see that at $\tau_{1}=0$, system (2.1) is stable focus but introducing $\tau_{2}=13.5$ induces limit cycle oscillations in the system (5.1). Next, we set $\tau_{1}=60$, where system (2.1) shows limit cycle oscillations, we see that at $\tau_{2}=8$, system (5.1) enters into chaotic regime. Finally, we set $\tau_{1}=139$, where system (2.1) exhibits chaotic dynamics, we see that system (5.1) shows extinction of $P$. volitans populations at $\tau_{2}=5$.

\section{Conclusions}

In this paper, we proposed a stage-structured prey-predator model with stagestructure of predator only. We considered cannibalism between adult and juvenile predator and a non-constant harvesting policy of the adult predator. To make the system more realistic, we considered delay in the growth rate of the juvenile preda- 

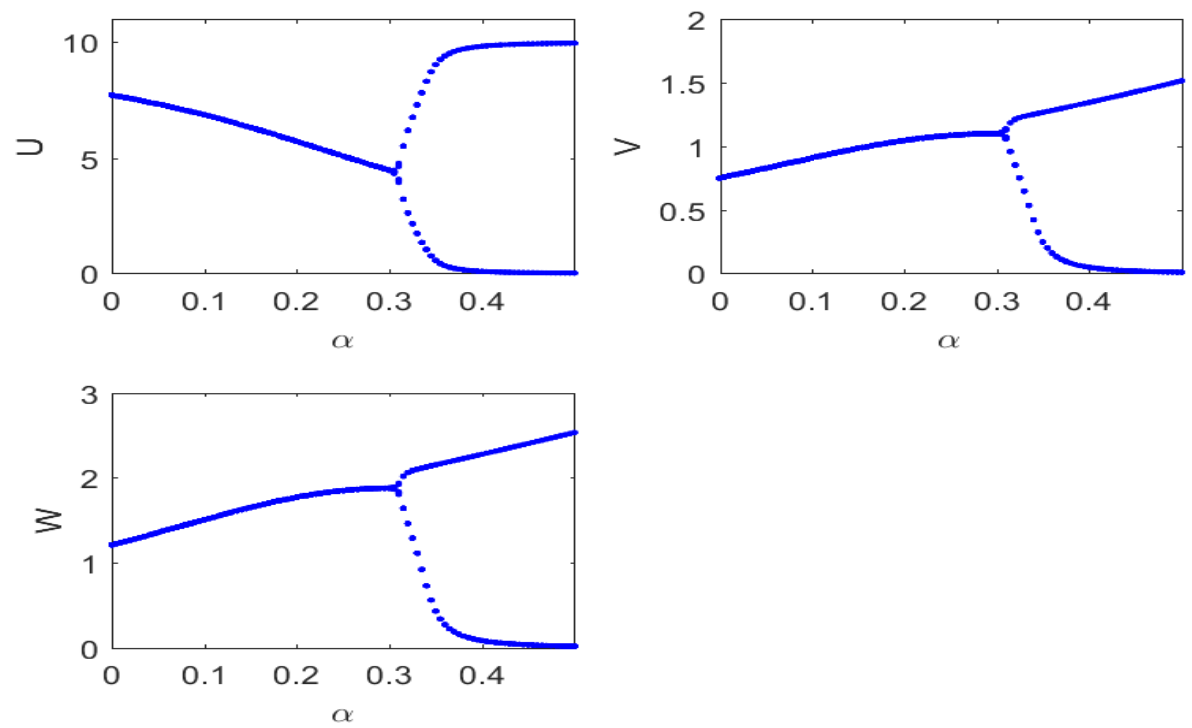

Fig. 8. Bifurcation diagram of the system (3.1) with respect to $\alpha$. Rest of the parameter values are same as in Table 1.

tor. The predation rate of the adult predator on prey population was assumed to follow modified Beddington type interactions. We analyze the model and explore the rich dynamics of the system in the absence and presence of the time delay. Firstly, the equilibrium and stability analysis have been done to find the possibility for stable coexistence of both prey and predator populations. Of the three feasible equilibria, the population-free equilibrium $E_{0}$ is unstable, only the $P$. volitans-free equilibrium $E_{1}$ and coexistence equilibrium $E^{*}$ can be attained by the system. A sufficient condition for the local asymptotic stability of the equilibrium $E_{1}$ imply that with high harvesting rate (or low predation rate) of adult $P$. volitans, the system stabilizes at the equilibrium $E_{1}$. If instead harvesting rate is low (or predation rate is high), $P$. volitans-free equilibrium becomes unstable, then coexistence occurs, either at equilibrium $E^{*}$ or through persistent oscillations of all the system's populations. Under suitable conditions, the coexistence point is shown to be globally asymptotically stable. The stability and bifurcations affecting the stable coexistence of prey and their predator are obtained analytically and supported by numerical simulations. PRCC values of parameters of interest with these organisms show that increasing the rate of harvesting of the adult $P$. volitans or decreasing the rate of predation of $P$. volitans on Parrotfish or decreasing the rate of cannibalism may be beneficial to the growth of Parrotfish in the system. As the Parrotfish feed on the algae, loss of herbivore causes a huge spread in algal biomass over the coral reefs, which in turn slows the growth of corals. 

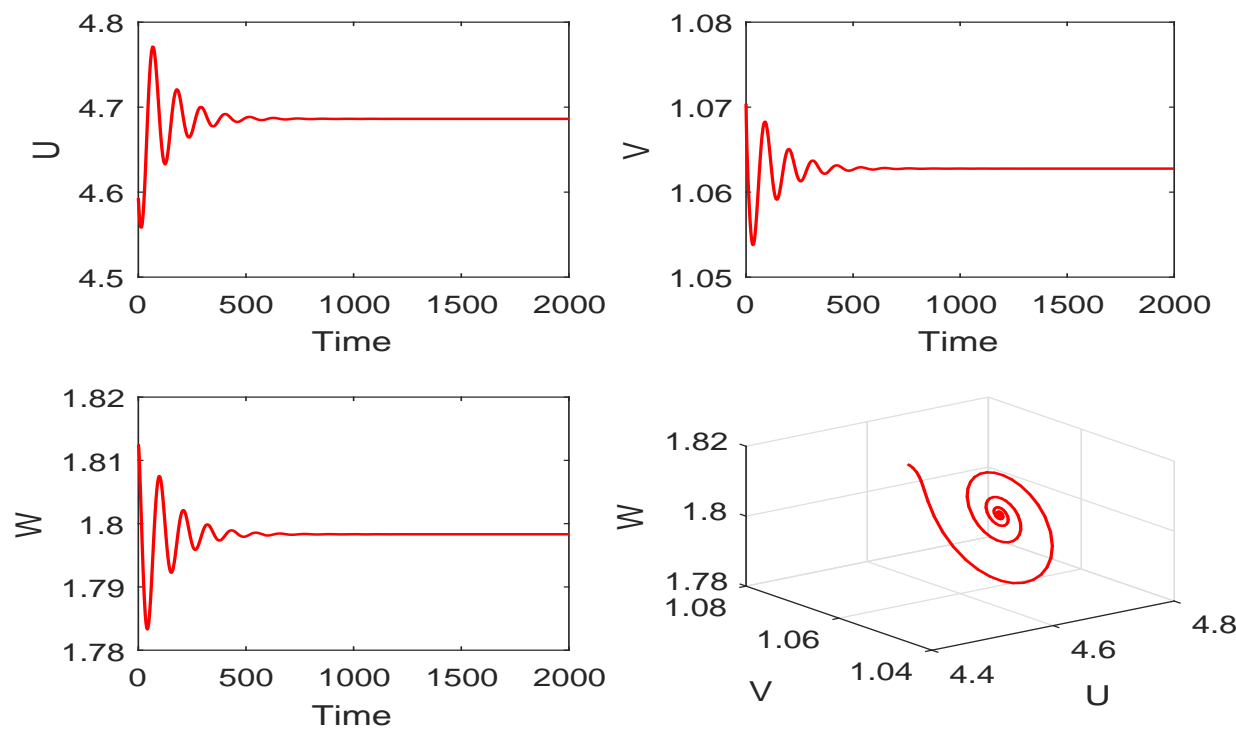

Fig. 9. System (2.1) shows stable focus at $\tau=1$. Rest of the parameter values are same as in Table 1 except $m_{1}=0.52$.

In the absence of time delay, we investigated how an increase in the predation rate of $P$. volitans on Parrotfish and the conversion efficiency due to cannibalism affected stability behaviour of the system. We found that the $P$. volitans-free and coexistence equilibria of the system are related via transcritical bifurcation with the predation rate of $P$. volitans on Parrotfish as a bifurcation parameter. The former losses its stability and the latter arises when the parameter crosses its critical value from below. Moreover, high rate of invasion of adult P. volitans on Parrotfish induces oscillations around the positive equilibrium, leading to dynamic instability. This represents the phenomenon of ecological imbalance due to the presence of the invasive $P$. volitans in a coral reef ecosystem, justifying the observations of Albins and Hixon ${ }^{20}$ that high predation rates of adult $P$. volitans are detrimental to coral reef ecosystems. Also, with high values of conversion efficiency due to cannibalism, the coexisting populations show oscillatory dynamics, which supports the observations from previous modeling analyses by Diekmann et al. ${ }^{58}$, Hastings ${ }^{59}$, and Magnusson ${ }^{60}$ that high cannibalism level can have destabilizing effects leading to oscillations. Coral reef ecosystems are subjected to a large number of factors influencing its dynamics. However, when or where the lionfish density is particularly high, the controlled harvesting of adults could be one of the most useful method to preserve or favor the reef ecosystems equilibrium as it allows an effective macroalgae grazing by native fish populations ${ }^{61}$.

In the study of Bhattacharya and $\mathrm{Pal}^{33}$, it is shown that the system undergoes 

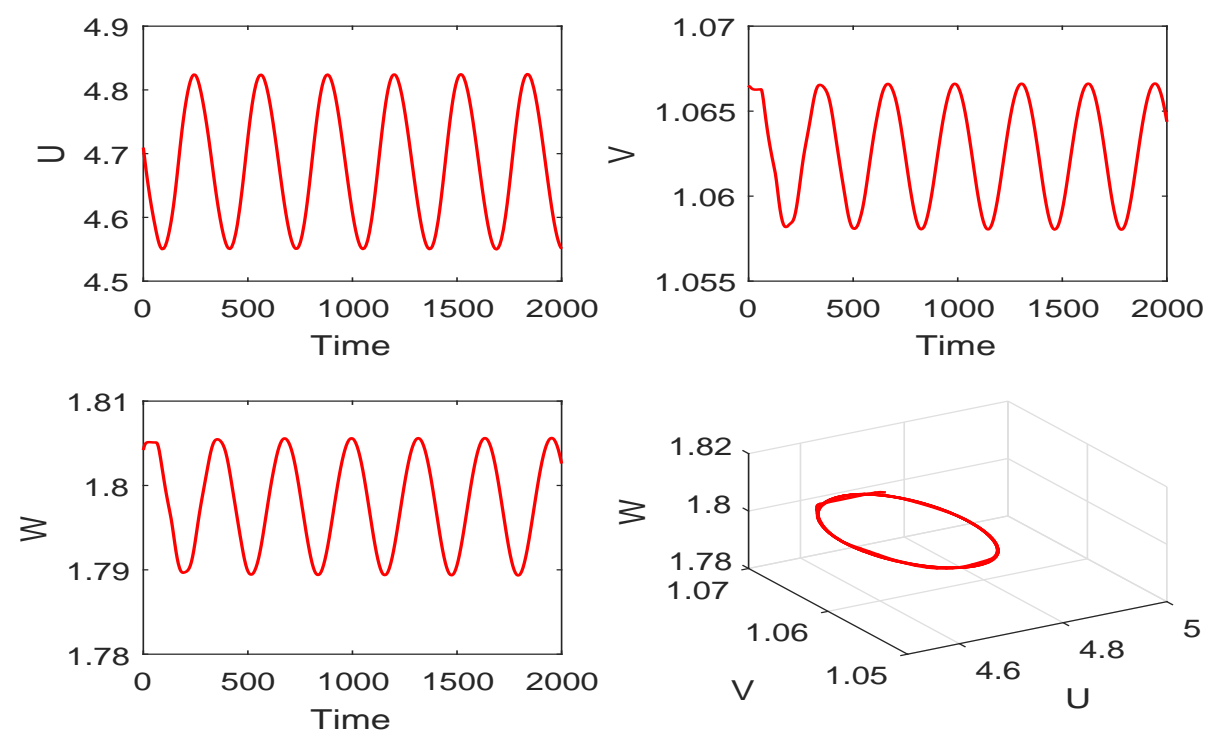

Fig. 10. System (2.1) shows limit cycle oscillations at $\tau=60$. Rest of the parameter values are same as in Table 1 except $m_{1}=0.52$.

limit cycle oscillations when the time lags (due to reproduction and maturation) cross certain critical values. In the present investigation, we found that with an increase in the values of time delay, the system undergoes chaotic dynamics through period doubling oscillations. To find the expression for various threshold values, we have plotted the bifurcation diagram of the system by varying the time delay. In order to confirm the occurrence of chaos, we drew the Poincaré map and computed the Lyapunov exponents. From ecological point of view, chaos has great biological importance to the system dynamics. Many theoretical studies reveal that ecosystem features such as predictability, species persistence ${ }^{62}$ and bio-diversity ${ }^{40}$ can be affected by chaos. In our model system, we observe that longer delay in the growth of juvenile $P$. volitans population is responsible for chaotic behavior of the system. The main reason behind the occurrence of this situation is that as the delay in the growth of juvenile $P$. volitans population increases, the growth of the adult population declines, which leads to a rapid growth of Parrotfish population in the system. This might be a benefit to the aquatic system as Parrotfish balance the growth of macroalgae. We have also seen the effect of time delay in an oscillating system. We observed that time delays increases the period and amplitude of oscillations if the system is already oscillatory but for large values of time delay the $P$. volitans populations extinct from the system. Further, we have seen the effects of reproduction delay as well as maturation delay in the system. To observe the effect of maturation delay, we choose the values of reproduction delay from the stable, un- 

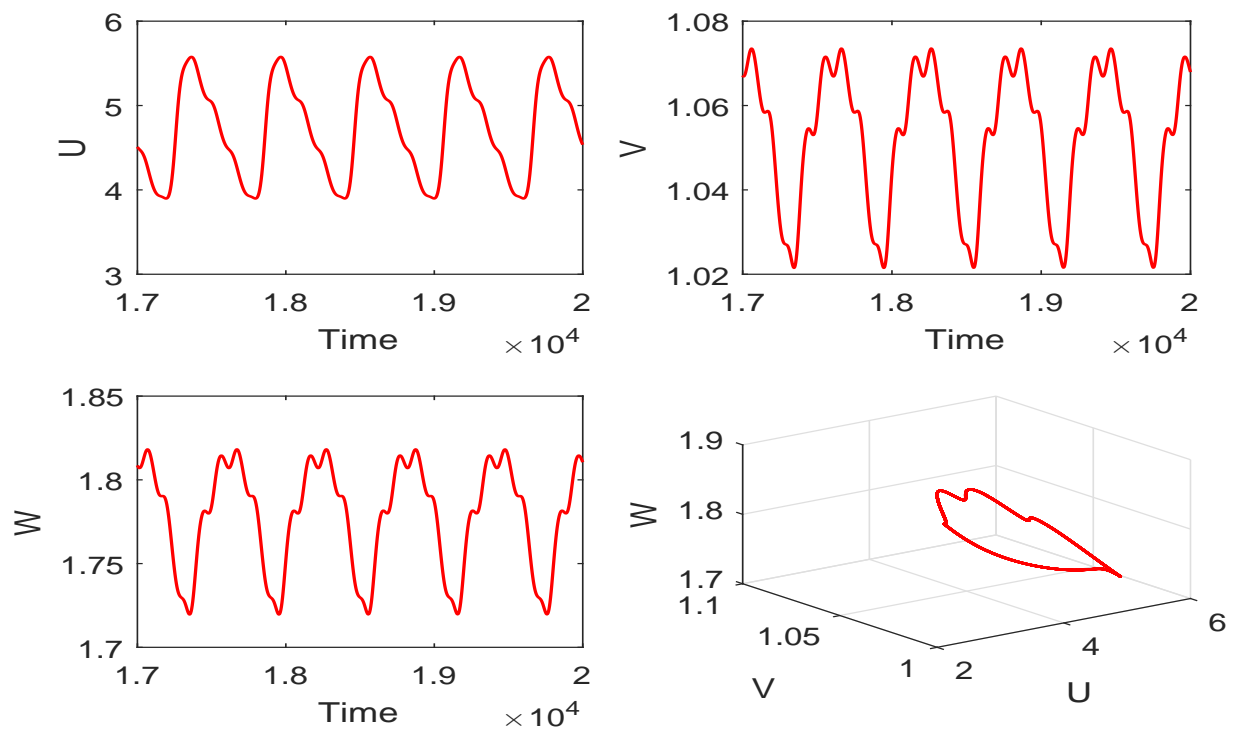

Fig. 11. System (2.1) shows period doubling oscillations at $\tau=125$. Rest of the parameter values are same as in Table 1 except $m_{1}=0.52$.

stable and chaotic regions. We observed that maturation delay induced limit cycle oscillations whenever reproduction delay has stabilizing effect. But, if the system is oscillatory due to reproduction delay, in that case system exhibits chaotic dynamics due to maturation delay. Further, maturation delay drives the system to the extinction of $P$. volitans populations if the system is chaotic due to reproduction delay. We hope that the findings of this article will certainly help the ecologists and as a consequence, it may enrich theoretical ecology.

\section{Acknowledgement}

The authors are grateful to the associate editor and anonymous referees for their careful reading, valuable comments and helpful suggestions, which have helped us to improve the presentation of this work significantly. Authors are also grateful to Prof. Guido Badino, DBIOS, University of Turin, Italy for his valuable suggestions. Pankaj Kumar Tiwari is thankful to University Grants Commissions, New Delhi, India for providing financial support in form of D. S. Kothari post-doctoral fellowship (No.F.4-2/2006 (BSR)/MA/17-18/0021).

\section{References}

1. Zhang X, Chen L, Neumann AU, The stage-structured predator-prey model and optimal harvesting policy, Math Biosci 168(2):201-210, 2000. 

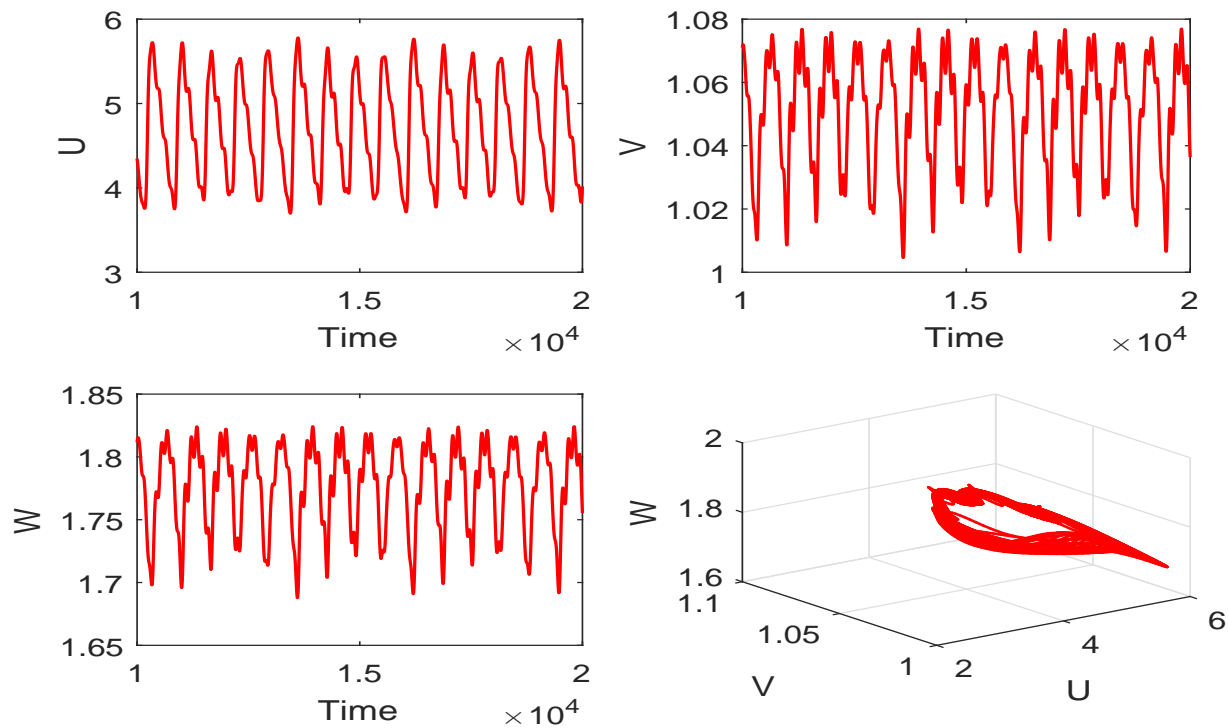

Fig. 12. System (2.1) shows chaotic dynamics at $\tau=139$. Rest of the parameter values are same as in Table 1 except $m_{1}=0.52$.

2. Wang W, Chen L, A predator-prey system with stage structure for predator, Comp Math Appl 33(8):83-91, 1997.

3. Rudolf VHW, The interaction of cannibalism and omnivory: consequences for community dynamics, Ecology 88:2697-2705, 2007.

4. Rudolf VHW, The influence of size-specific indirect interactions in predator-prey systems, Ecology 87:362-371, 2006.

5. Rudolf VHW, The impact of cannibalism in the prey on predator-prey dynamics, Ecology 89:3116-3127, 2008.

6. Lili J, Chengqiang W, Qualitative analysis of a predator-prey model with constant-rate prey harvesting incorporating a constant prey refuge, Nonlinear Anal RWA 11:22852295, 2010.

7. Chen L, Chen F, Chen L, Qualitative analysis of a predator-prey model with Holling type II functional response incorporating a constant prey refuge, Nonlinear Anal RWA 11:246-252, 2010.

8. Jost C, Arino O, Arditi R, About deterministic extinction in ratio-dependent predatorprey models, Bull Math Biol 61:19-32, 1999.

9. Kuang Y, Rich dynamics of Gause-type ratio-dependent predator prey system, Fields Instit Commun 21:325-337, 1999.

10. Xiao D, Ruan S, Global dynamics of a ratio-dependent predator-prey system, $J$ Math Biol 43:268-290, 2001.

11. Hsu SB, Hwang TW, Kuang Y, Global analysis of the Michaelis-Menten type ratiodependent predator-prey system, J Math Biol 42(6):489-506, 2001.

12. Akcakaya HR, Arditi R, Ginzburg LR, Ratio-dependent prediction: an abstraction that works, Ecology 76:9951004, 1995.

13. Morris Jr JA et al., Biology and ecology of invasive lionfishes, Pterois miles and Pterois 

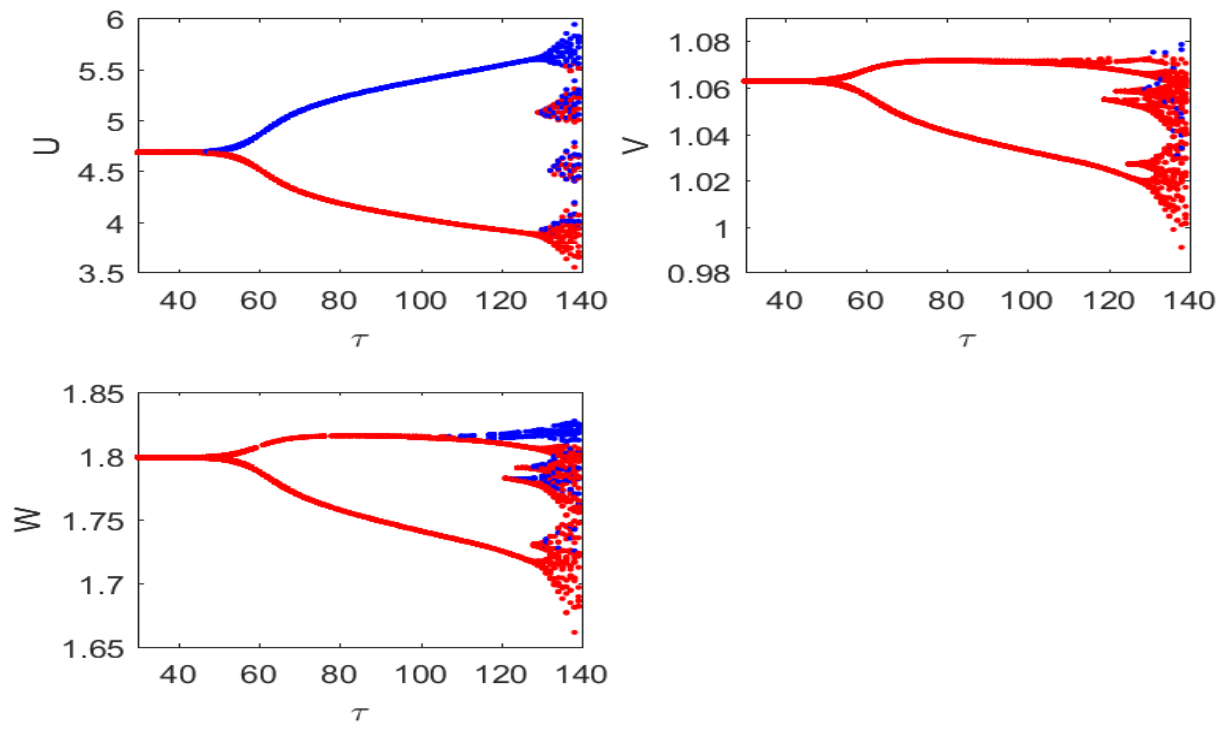

Fig. 13. Bifurcation diagram of the system (2.1) with respect to $\tau$. Here, the maximum and minimum values of the oscillations are plotted in blue and red colors, respectively. Rest of the parameter values are same as in Table 1 except $m_{1}=0.52$.

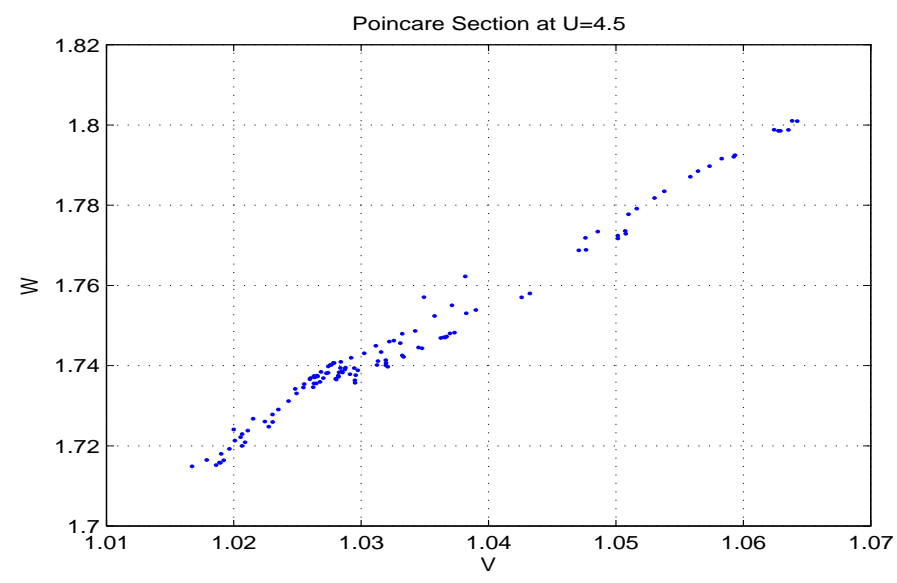

Fig. 14. Poincaré map of the system (2.1) in $V-W$ plane at $U=4.5$ for $\tau=139$. Rest of the parameter values are same as in Table 1 except $m_{1}=0.52$.

volitans, Gulf and Caribbean Fisheries Institute 61:1-6, 2009.

14. Chagaris D et al., An ecosystem-based approach to evaluating impacts and management of invasive lionfish, Fisheries 42(8):422-431, 2017. 


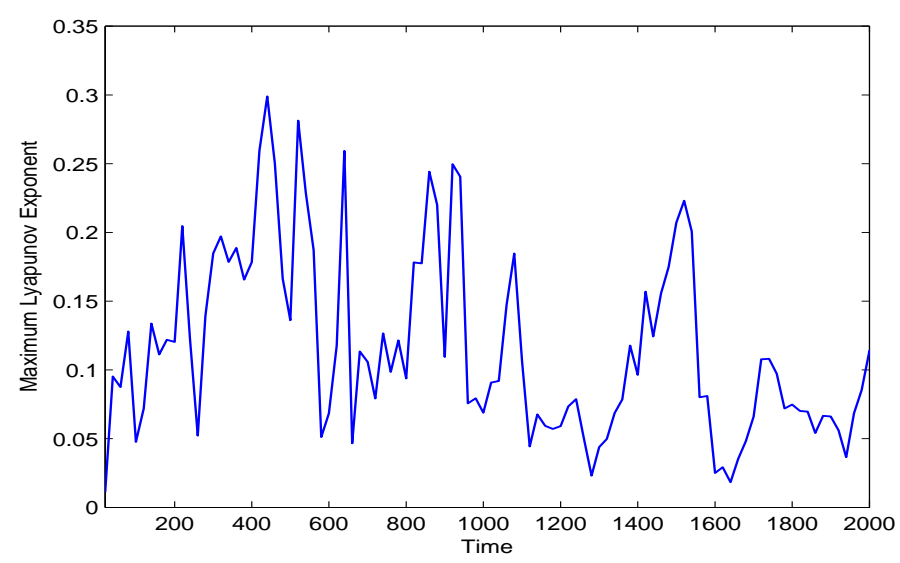

Fig. 15. The maximum Lyapunov exponent of the system (2.1) for $\tau=139$. Rest of the parameter values are same as in Table 1 except $m_{1}=0.52$.

15. Fogg AQ, Life history of the non-native invasive red lionfish (Pterois volitans) in the Northern Gulf of Mexico, The University of Southern Mississippi, ProQuest Dissertations Publishing 10266372:164 pages, 2017.

16. Peake $\mathrm{J}$ et al., Feeding ecology of invasive lionfish (Pterois volitans and Pterois miles) in the temperate and tropical western Atlantic, Biol Invasions 20:2567-2597, 2018.

17. Fishelson L, Experiments and observations on food consumption growth and starvation in Dendrochirus brachypterus and Pterois volitans (Pteroinae, Scorpaenidae), Environ Biol Fishes 50:391-403, 1997.

18. Ludwig D, Jones DD, Holling CS, Qualitative analysis of insect outbreak systems: the spruce budworm and forest, J Anim Ecol 47(1):315-332, 1978.

19. Kindinger TL, Albins MA, Consumptive and non-consumptive effects of an invasive marine predator on native coral-reef herbivors, Biol Invasions 19:131-146, 2017.

20. Albins MA, Hixon MA, Invasive Indo-Pacific lionfish (Pterois volitans) reduce recruitment of Atlantic coral-reef fishes, Mar Ecol Pro Ser 367:233-238, 2008.

21. Benkwitt CE, Nonlinear effects of invasive lionfish density on native coral-reef fish communities, Biol Invasions 17:1383-1395, 2015.

22. Hare JA, Whitfield PE, An integrated assessment of the introduction of lionfish (Pterois Volitans/miles complex) to the western Atlantic Ocean, NOAA Technical Memorandum NOS NCCOS 2:1-21, 2003.

23. Goreau TJ, Hayes R, Coral bleaching and ocean "hot spots", Ambio 23:176-180, 1994.

24. Bhattacharyya J, Pal S, Hysteresis in coral reefs under macroalgal toxicity and overfishing, J Biol Phys 41(2):151-172, 2015.

25. Rotjan RD, Lewis SM, Parrotfish abundance and selective corallivory on a Belizean coral reef, J Exper Mar Biol Ecol 335:292-301, 2006.

26. Morris JAJr, Whitfield PE, Biology, ecology, control and management of the invasive Indo-Pacific lionfish: an updated integrated assessment, NOAA Technical Memorandum NOS-NCCOS 99:1-65, 2009.

27. Bhattacharyya J, Pal S, The role of space in stage-structured cannibalism with harvesting of an matured predator, Comput Math Appl 66:339355, 2013.

28. Bhattacharya J, Pal S, Stage-structured cannibalism in a ratio-dependent system 

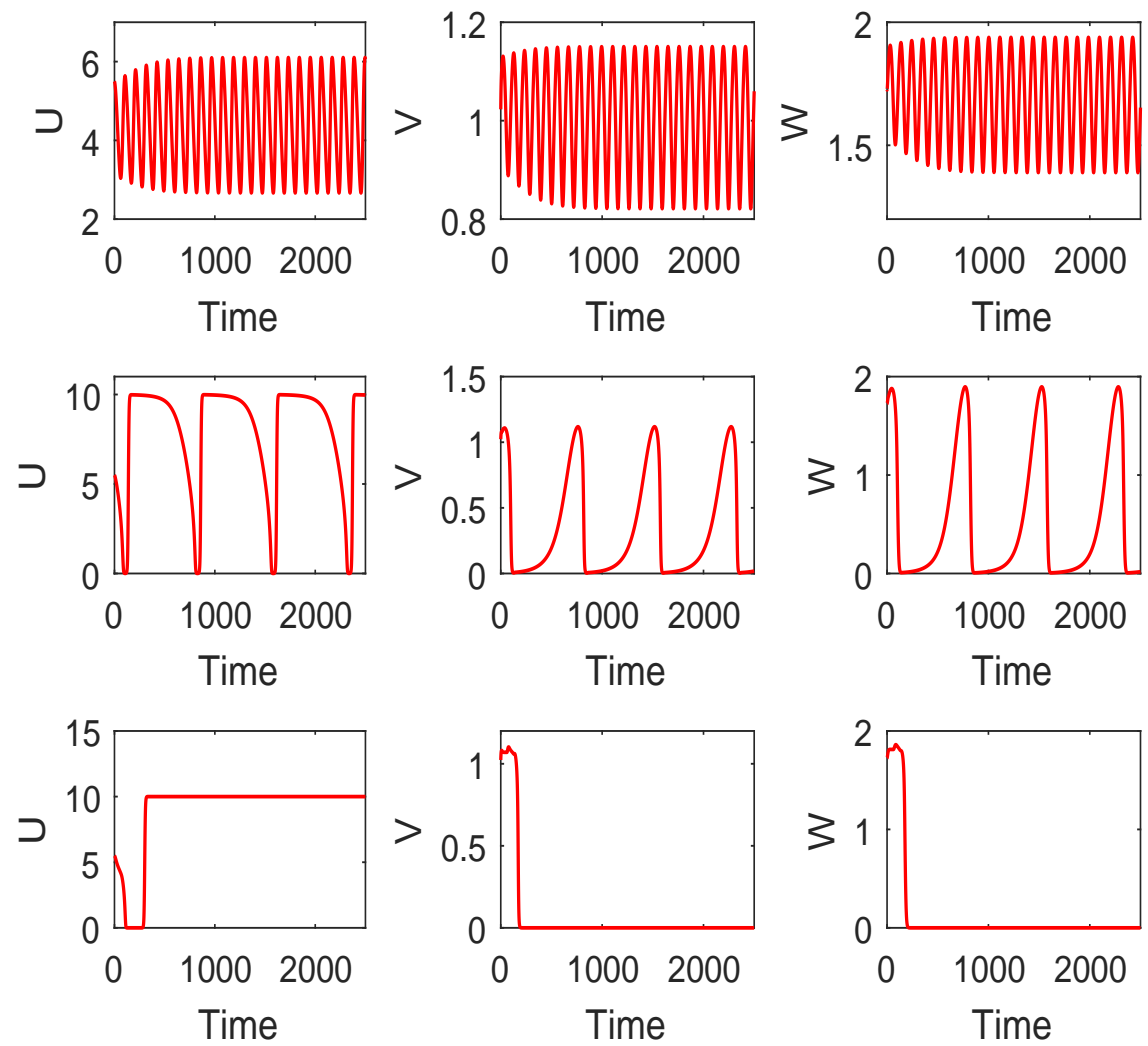

Fig. 16. Effect of time delay in an oscillating system $\left(m_{1}=0.53\right): \tau=0$ (first row), $\tau=10$ (second row), and $\tau=50$ (third row). Rest of the parameter values are same as in Table 1 .

with constant prey refuge and harvesting of matured predator, Differ Equ Dyn Syst 24(3):345-366, 2016.

29. Biswas S, Samanta S, Chattopadhyay J, Cannibalistic predator-prey model with disease in predator - A delay model, Int J Bifur Chaos 25(10):1550130, 2015.

30. Gopalsamy K, Stability and oscillations in delay differential equations of population dynamics, Mathematics and its applications, Kluwer Academic Pub Dordrecht 74, 1992.

31. Ruan S, Wei J, Stability and bifurcation in a neural network model with two delays, Physica D 130:255-272, 1999.

32. Ruan S, Wei J, On the zeros of transcendental functions with applications to stability of delay differential equations with two delays, Dyn Contin Disc Impul Syst Ser A Math Anal 10:863-874, 2003.

33. Bhattacharya J, Pal S, Dynamics of asymmetric intraguild predation with time lags in reproduction and maturation, Cogent Math 2:1021604, 2015. 


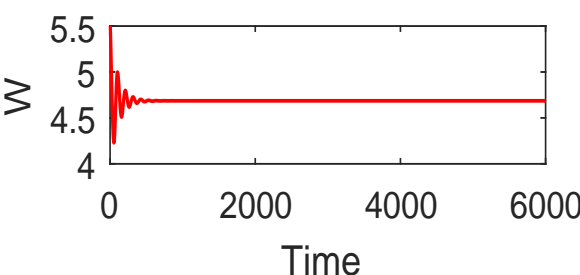

(a)

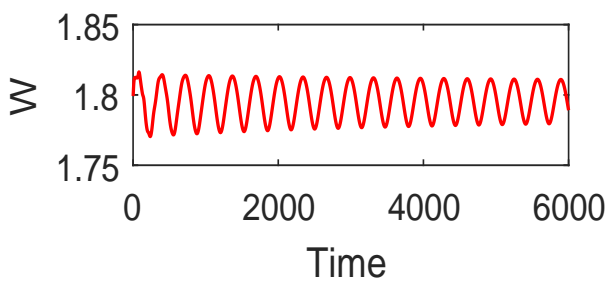

(c)

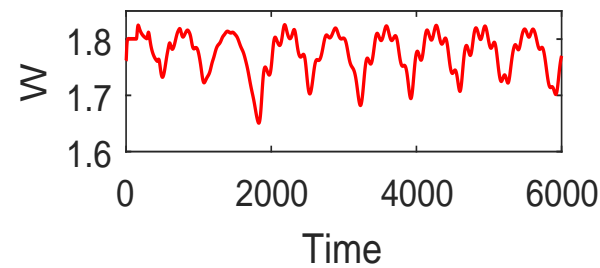

(e)

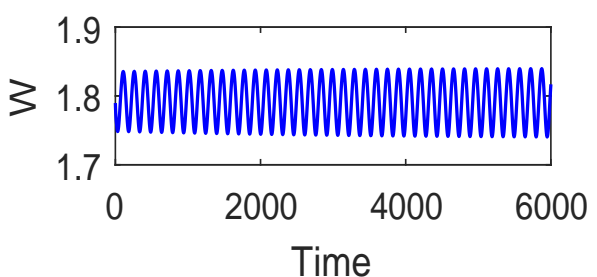

(b)

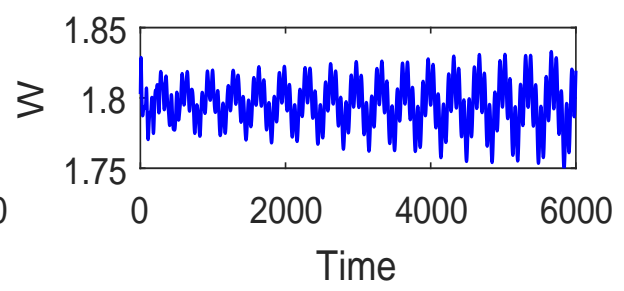

(d)

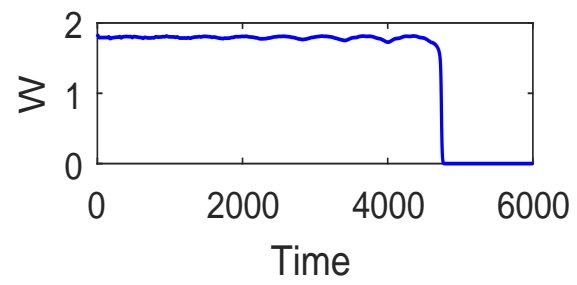

(f)

Fig. 17. Effects of reproduction delay $\left(\tau_{1}\right)$ and maturation delay $\left(\tau_{2}\right)$ in the system (5.1): (a) $\tau_{1}=0$ and $\tau_{2}=0$, (b) $\tau_{1}=0$ and $\tau_{2}=13.5$, (c) $\tau_{1}=60, \tau_{2}=0$, (d) $\tau_{1}=60$ and $\tau_{2}=8$, (e) $\tau_{1}=139$ and $\tau_{2}=0$, and (f) $\tau_{1}=139$ and $\tau_{2}=5$. Rest of the parameter values are same as in Table 1 except $m_{1}=0.52$.

34. Bhattacharya J, Pal S, Dynamics of a stage-structured system with harvesting and discrete time delays, Syst Sci Cont Eng 2:192-215, 2014.

35. Chen G, Ueta T, Yet another chaotic attractor, Int J Bifur Chaos 9:1465-1466, 1999.

36. Gakkhar S, Naji R, Chaos in three species ratio-dependent food chain, Chaos Solit Fract 14:771-778, 2002.

37. Chattopadhayay J, Sarkar R, Chaos to order: preliminary experiments with a population dynamics models of three tropic levels, Ecol Model 163:45-50, 2003.

38. Hastings A, Powell T, Chaos in three-species food chain, Ecology 72:896-903, 1991.

39. May R, Biological populations with nonoverlapping generations: stable points, stable cycles, and chaos, Science 186:645-647, 1974.

40. Huisman J, Weissing F, Biodiversity of plankton by species oscillations and chaos, Nature 402,407-411, 1999. 
41. Turchin P, Complex population dynamics: a theoretical/empirical synthesis, Princeton: Princeton Univ. Press, 2003.

42. Nychka D et al., Finding chaos in noisy systems, J R Stat Soc B 54:399-426, 1992.

43. Ellner S, Turchin P, Chaos in a noisy world: new methods and evidence from timeseries analysis, Am Nat 145:343-375, 1995.

44. Becks L et al., Experimental demonstration of chaos in a microbial food web, Nature 435:1226-1229, 2005.

45. Beddington J, Mutual interference between parasites or predators and its effect on searching efficiency, J Anim Ecol 51:597-624, 1975

46. Leard B, Lewis C, Rebaza J, Dynamics of ratio-dependent predator-prey models with nonconstant harvesting, Dis Cont Dyn Syst Ser S 1(2):303-315, 2008.

47. Lenzini P, Rebaza J, Nonconstant predator harvesting on ratio-dependent predatorprey models, Appl Math Sci 4(13-16):791-803, 2010.

48. Castillo-Chavez $\mathrm{C}$ et al., Mathematical approaches for emerging and reemerging infectious diseases: An introduction, Vol. 126 of the IMA volumes in mathematics and its applications. New York, NY: Springer, 2002.

49. Martin A, Ruan S, Predator-prey models with delay and prey harvesting, J Math Biol 43:247-267, 2001.

50. Rudolf V, Antonovics J, Disease transmission by cannibalism: Rare event or common occurrence? Proc Roy Soc B 274:1205-1210, 2007.

51. Freedman HI, Ruan S, Tang M, Uniform persistence and flows near a closed positively invariant set, J Dyn Diff Equ 6:583-600, 1994.

52. Smith H, Paul W, The theory of the chemostat: Dynamics of microbial competition, Cambridge University Press, Cambridge, 1994.

53. Castillo-Chavez C, Song B, Dynamical models of tuberculosis and their applications, Math Biosci Eng 1:361-404, 2004.

54. Blower SM, Dowlatabadi H, Sensitivity and uncertainty analysis of complex models of disease transmission: an HIV model, as an example, Int Stat Rev 62:229-243, 1994.

55. Marino S, Hogue IB, Ray CJ, Kirschner DE, A methodology for performing global uncertainty and sensitivity analysis in systems biology, $J$ Theor Biol 254(1):178-196, 2008.

56. Park T, A matlab version of the lyapunov exponent estimation algorithm of wolf et al. physica 16d, 1985, 2014. https://www.mathworks.com/matlabcentral/fileexchange/48084lyapunov-exponent-estimation-from-a-time-series-documentation-added.

57. Wolf A, Swift JB, Swinney HL, Vastano JA, Determining Lyapunov exponents from a time series, Physica D 16:285-317, 1985.

58. Diekmann O et al., Simple mathematical models for cannibalism: A critique and a new approach, Math Biosci 78:21-46, 1986.

59. Hastings A, Cycles in cannibalistic egglarval interactions, $J$ Math Biol 24:651-666, 1987.

60. Magnusson KG, Destabilizing effect of cannibalism on a structured predator-prey system, Math Biosci 155:61-75, 1999.

61. Hackerott $\mathrm{S}$ et al., Invasive lionfish had no measurable effect on prey fish community structure across the Belizean Barrier Reef, Peer J 5:e3270, 2017.

62. Allen JC, Schaffer WM, Rosko D, Chaos reduces species extinctions by amplifying local population noise, Nature 364:229-232, 1993.

63. Birkhoff G, Rota GC, Ordinary Differential Equations, 4th edn. Wiley, Ginn, Boston 1989.

64. Nagumo N, Uber die Lage der Integralkurven gewonlicher Dierantialgleichungen, Proc 
Phys Math Soc Jpn 24:551, 1942.

65. Li Y, Muldowney JS, On Bendixson's criterion, J Diff Eqn 106:27-39, 1993.

66. Poore AB, On the theory and application of the Hopf-Friedrichs bifurcation theory, Arch Rational Mech Anal 60(4):371-393, 1976.

\section{Appendix A}

Proof of Theorem 4.1 is as follows.

By setting $X=(U, V, W)^{T} \in \mathbb{R}^{3}$ and $F(X)=\left[F_{1}(X), F_{2}(X), F_{3}(X)\right]^{T}$, with $F: C \rightarrow \mathbb{R}^{3}$ and $F \in C^{\infty}\left(\mathbb{R}^{3}\right)$, equation (3.1) becomes

$$
\dot{X}=F(X),
$$

together with $X(0)=X_{0} \in \mathbb{R}_{+}^{3}$.

The vector function $F$ is a locally Lipschitzian and completely continuous function of variables $(U, V, W)$ in

$$
\Theta=\{(U(t), V(t), W(t)): U>0, V>0, W>0\},
$$

then any solution $(U, V, W)$ of system (3.1) with initial conditions (3.2) exists and unique in some interval $[0, \kappa) 63$.

It is easy to check that whenever choosing $X(0) \in \mathbb{R}_{+}^{3}$ with $X_{i}=0$ for $i=1,2,3$, then $\left.F_{i}(X)\right|_{X_{i}=0} \geq 0$. Due to lemma of Nagumo ${ }^{64}$, any solution of system (6.1) with $X_{0} \in \mathbb{R}_{+}^{3}$, say $X(t)=X\left(t ; X_{0}\right)$ is such that $X(t) \in \mathbb{R}_{+}^{3}$ for all $t>0$.

Proof of Theorem 4.2 is as follows.

Let us define a new variable $P=U+\frac{1}{\alpha_{1}}(V+W)$. For an arbitrary $\eta>0$, by summing up the equations in system (3.1), we find

$$
\frac{d P}{d t}+\eta P=(r+\eta) U-\frac{r U^{2}}{K}-\frac{\left(D_{1}-\eta\right) V}{\alpha_{1}}-\frac{\left(D_{2}-\eta\right) W}{\alpha_{1}}-\frac{(1-\alpha) m_{2} V W}{\alpha_{1}\left(a_{2}+V\right)}-\frac{h W}{\alpha_{1}(c+W)} .
$$

After choosing $\eta \leq \min \left\{D_{1}, D_{2}\right\}$, we obtain the following upper bound:

$$
\frac{d P}{d t}+\eta P \leq(r+\eta) U-\frac{r U^{2}}{K} \leq \frac{K(r+\eta)^{2}}{4 r}=L
$$

Applying standard results on differential inequalities, we have

$$
P(t) \leq e^{-\eta t}\left(P(0)-\frac{L}{\eta}\right)+\frac{L}{\eta} \leq \max \left\{\frac{L}{\eta}, P(0)\right\}=M .
$$

Thus, there exists an $M>0$, depending only on the parameters of system (3.1), such that $P(t)<M$ for all $t$ large enough. Hence, the solutions of system (3.1) and consequently all the densities appearing in the system are ultimately bounded above $^{63}$.

Proof of Theorem 4.3 is as follows.

Since $\lim _{t \rightarrow \infty} \sup \left[U(t)+\frac{1}{\alpha_{1}}(V(t)+W(t))\right] \leq M$, it follows that $\lim _{t \rightarrow \infty}[V(t)+$ $W(t)] \leq M$. Therefore, there exists $T_{1}>0$ such that $V(t) \leq M_{1}$ and $W(t) \leq M_{2}$ for all $t \geq T_{1}$, where $M_{1}$ and $M_{2}$ are finite positive constants with $M_{1}+M_{2}<M$. 
Also, $U(t) \leq K$ as $t \rightarrow \infty$. Therefore, there exists $T_{2}>0$ such that $U(t) \leq K$ for all $t \geq T_{2}$. For all $t \geq \max \left\{T_{1}, T_{2}\right\}$, we have $\frac{d U}{d t} \geq r U\left(1-\frac{U}{K}\right)-\frac{m_{1} U}{a_{1}}$. This implies $\left.\frac{d U}{d t}\right|_{u_{1}} \geq 0$ for all $t \geq \max \left\{T_{1}, T_{2}\right\}$, where $u_{1}=K\left(1-\frac{m_{1}}{r a_{1}}\right)>0$ and $r>\frac{m_{1}}{a_{1}}$. Therefore, there exists $0<u_{1} \leq K$ such that $u_{1} \leq U(t) \leq K$.

For $t>\max \left\{T_{1}, T_{2}\right\}$, we have

$$
\begin{aligned}
\frac{d V}{d t} & \geq \frac{\alpha_{1} m_{1} u_{1} W}{a_{1} M_{2}+b_{1} K+c_{1}}-\left(\mu+D_{1}\right) M_{1}-(1-\alpha) m_{2} W \\
& >0 \text { if } W(t)>\frac{\left(a_{1} M_{2}+b_{1} K+c_{1}\right)\left(\mu+D_{1}\right) M_{1}}{\alpha_{1} m_{1} u_{1}-(1-\alpha) m_{2}\left(a_{1} M_{2}+b_{1} K+c_{1}\right)} \text { and } u_{1}>\frac{m_{2}(1-\alpha)\left(a_{1} M_{2}+b_{1} K+c_{1}\right)}{\alpha_{1} m_{1}} .
\end{aligned}
$$

Thus, for all $t>\max \left\{T_{1}, T_{2}\right\}$, if $u_{1}>\frac{m_{2}(1-\alpha)\left(a_{1} M_{2}+b_{1} K+c_{1}\right)}{\alpha_{1} m_{1}}$, then there exists $w_{1}>0$ such that $\frac{M_{1}\left(a_{1} M_{2}+b_{1} K+c_{1}\right)\left(\mu+D_{1}\right)}{\alpha_{1} m_{1} u_{1}-(1-\alpha) m_{2}\left(a_{1} M_{2}+b_{1} K+c_{1}\right)}<w_{1}<M_{2}$.

Therefore, for all $t>\max \left\{T_{1}, T_{2}\right\}, \frac{d V}{d t}>0$ for $W(t) \geq w_{1}>0$ and so in this case there exists $T_{3}>0$ and $0<v_{1}<M_{1}$ such that $V(t) \geq v_{1}$ for all $t \geq T_{3}$. Also, for all $t>\max \left\{T_{1}, T_{2}, T_{3}\right\}$,

$$
\frac{d W}{d t} \geq \mu v_{1}-D_{2} M_{2}-h>0 \text { if } v_{1}>\frac{D_{2} M_{2}+h}{\mu} .
$$

Therefore, for large values of $t$, if $r>\frac{m_{1}}{a_{1}}$ holds, then there exist $u_{1}>K\left(1-\frac{m_{1}}{r a_{1}}\right), v_{1}>\frac{1}{\mu}\left(D_{2} M_{2}+h\right), w_{1}>\frac{M_{1}\left(\mu+D_{1}\right)\left(a_{1} M_{2}+b_{1} K+c_{1}\right)}{\alpha_{1} m_{1} u_{1}-m_{2}(1-\alpha)\left(a_{1} M_{2}+b_{1} K+c_{1}\right)}$ such that

$$
u_{1} \leq U(t) \leq K, v_{1} \leq V(t) \leq M_{1}, w_{1} \leq W(t) \leq M_{2}
$$

\section{Appendix B}

Jacobian of system (3.1) is given by

$$
J=\left(\begin{array}{ccc}
J_{11} & 0 & J_{13} \\
J_{21} & J_{22} & J_{23} \\
0 & J_{32} & J_{33}
\end{array}\right)
$$

where

$$
\begin{aligned}
& J_{11}=r\left(1-\frac{2 U}{K}\right)-\frac{m_{1} W\left(a_{1} W+c_{1}\right)}{\left(a_{1} W+b_{1} U+c_{1}\right)^{2}}, J_{13}=-\frac{m_{1} U\left(b_{1} U+c_{1}\right)}{\left(a_{1} W+b_{1} U+c_{1}\right)^{2}}, J_{21}=\frac{\alpha_{1} m_{1} W\left(a_{1} W+c_{1}\right)}{\left(a_{1} W+b_{1} U+c_{1}\right)^{2}}, \\
& J_{22}=-\left(\mu+D_{1}+\frac{m_{2} a_{2}(1-\alpha) W}{\left(a_{2}+V\right)^{2}}\right), J_{23}=\frac{\alpha_{1} m_{1} U\left(b_{1} U+c_{1}\right)}{\left(a_{1} W+b_{1} U+c_{1}\right)^{2}}-\frac{(1-\alpha) m_{2} V}{a_{2}+V} \\
& J_{32}=\mu, J_{33}=-\left(D_{2}+\frac{h c}{(c+W)^{2}}\right) .
\end{aligned}
$$


1. Jacobian $J$ evaluated at the equilibrium $E_{0}$ leads to the eigenvalues $r,-\left(\mu+D_{1}\right)$ and $-\left(D_{2}+\frac{h}{c}\right)$. Since one eigenvalue is always positive, the equilibrium $E_{0}$ is unconditionally unstable.

2. Jacobian $J$ evaluated at the equilibrium $E_{1}$ immediately gives one eigenvalue $-r$ and two eigenvalues as roots of the quadratic

$$
\lambda^{2}+\left(\mu+D_{1}+D_{2}+\frac{h}{c}\right) \lambda+\left(\mu+D_{1}\right)\left(D_{2}+\frac{h}{c}\right)-\frac{\mu \alpha_{1} m_{1} K}{b_{1} K+c_{1}}=0
$$

Define $m_{1}^{*}=\frac{\left(b_{1} K+c_{1}\right)\left(\mu+D_{1}\right)}{\mu \alpha_{1} K}\left(D_{2}+\frac{h}{c}\right)$. Then both roots of equation (6.2) are either negative or with negative real parts if $m_{1}<m_{1}^{*}$ and thus the equilibrium $E_{1}$ is stable. On the other hand, equation (6.2) has one positive and one negative roots if $m_{1}>m_{1}^{*}$ and in this case the equilibrium $E_{1}$ is unstable as one eigenvalue is positive.

3. The Jacobian $J$ evaluated at the equilibrium $E^{*}$ leads to the following matrix

$$
J_{E^{*}}=\left(\begin{array}{ccc}
-a_{11} & 0 & -a_{13} \\
a_{21} & -a_{22} & a_{23} \\
0 & a_{32} & -a_{33}
\end{array}\right)
$$

where

$$
\begin{aligned}
& a_{11}=\left(\frac{r U^{*}}{K}-\frac{m_{1} b_{1} U^{*} W^{*}}{\left(a_{1} W^{*}+b_{1} U^{*}+c_{1}\right)^{2}}\right), a_{13}=\frac{m_{1} U^{*}\left(b_{1} U^{*}+c_{1}\right)}{\left(a_{1} W^{*}+b_{1} U^{*}+c_{1}\right)^{2}}, a_{21}=\frac{\alpha_{1} m_{1} W^{*}\left(a_{1} W^{*}+c_{1}\right)}{\left(a_{1} W^{*}+b_{1} U^{*}+c_{1}\right)^{2}} \\
& a_{22}=\left(\mu+D_{1}+\frac{m_{2} a_{2}(1-\alpha) W^{*}}{\left(a_{2}+V^{*}\right)^{2}}\right), a_{23}=\frac{\alpha_{1} m_{1} U^{*}\left(b_{1} U^{*}+c_{1}\right)}{\left(a_{1} W^{*}+b_{1} U^{*}+c_{1}\right)^{2}}-\frac{(1-\alpha) m_{2} V^{*}}{a_{2}+V^{*}} \\
& a_{32}=\mu, a_{33}=\left(D_{2}+\frac{h c}{\left(c+W^{*}\right)^{2}}\right) .
\end{aligned}
$$

The associated characteristic equation is

$$
\lambda^{3}+A_{1} \lambda^{2}+A_{2} \lambda+A_{3}=0,
$$

where

$$
\begin{aligned}
& A_{1}=a_{11}+a_{22}+a_{33}, A_{2}=a_{11}\left(a_{22}+a_{33}\right)+a_{22} a_{33}-a_{23} a_{32}, \\
& A_{3}=a_{11}\left(a_{22} a_{33}-a_{23} a_{32}\right)+a_{13} a_{21} a_{32} .
\end{aligned}
$$

Using Routh-Hurwitz criterion, roots of equation (6.3) are either negative or have negative real parts if and only if the conditions in (4.2) are satisfied. 


\section{Appendix C}

Observe that one eigenvalue of the Jacobian matrix

$$
J\left(E_{1}, m_{1}^{*}\right)=\left(\begin{array}{ccc}
-r & 0 & -\frac{\mu+D_{1}}{\mu \alpha_{1}}\left(D_{2}+\frac{h}{c}\right) \\
0 & -\left(\mu+D_{1}\right) & \frac{\mu+D_{1}}{\mu}\left(D_{2}+\frac{h}{c}\right) \\
0 & \mu & -\left(D_{2}+\frac{h}{c}\right)
\end{array}\right)
$$

is $\lambda=0$. Hence, at $m_{1}=m_{1}^{*}$ the equilibrium $E_{1}$ is non-hyperbolic and the assumption (A1) of Theorem 4.1 in Castillo-Chavez and Song ${ }^{53}$ is verified.

Now, denote by $\mathbf{w}=\left(w_{1}, w_{2}, w_{3}\right)^{T}$ a right eigenvector associated with the zero eigenvalue $\lambda=0$. To determine the components of $\mathbf{w}$, we solve the following system of equations:

$$
\begin{aligned}
-r w_{1}-\frac{\mu+D_{1}}{\mu \alpha_{1}}\left(D_{2}+\frac{h}{c}\right) w_{3} & =0, \\
-\left(\mu+D_{1}\right) w_{2}+\frac{\mu+D_{1}}{\mu}\left(D_{2}+\frac{h}{c}\right) w_{3} & =0, \\
\mu w_{2}-\left(D_{2}+\frac{h}{c}\right) w_{3} & =0,
\end{aligned}
$$

to obtain $w_{1}=-\frac{\mu+D_{1}}{r \mu \alpha_{1}}\left(D_{2}+\frac{h}{c}\right) w_{3}$ and $w_{2}=\frac{1}{\mu}\left(D_{2}+\frac{h}{c}\right) w_{3}$.

Furthermore, the components of the left eigenvector $\mathbf{v}=\left(\mathbf{v}_{\mathbf{1}}, \mathbf{v}_{\mathbf{2}}, \mathbf{v}_{\mathbf{3}}\right)$ can be determined by solving the following system of equations:

$$
\begin{aligned}
-r v_{1} & =0, \\
-\left(\mu+D_{1}\right) v_{2}+\mu v_{3} & =0, \\
-\frac{\mu+D_{1}}{\mu \alpha_{1}}\left(D_{2}+\frac{h}{c}\right) v_{1}+\frac{\mu+D_{1}}{\mu}\left(D_{2}+\frac{h}{c}\right) v_{2}-\left(D_{2}+\frac{h}{c}\right) v_{3} & =0,
\end{aligned}
$$

to obtain $v_{1}=0$ and $v_{3}=\frac{\mu+D_{1}}{\mu} v_{2}$. We choose $w_{3}=\frac{\mu}{\mu+D_{1}+D_{2}+\frac{h}{c}}$ and $v_{2}=1$, so that $\mathbf{w} \cdot \mathbf{v}=1$.

Now, the coefficients $a$ and $b$ defined in Theorem 4.1 of Castillo-Chavez and Song 53

$$
a=\sum_{k, i, j=1}^{3} v_{k} w_{i} w_{j} \frac{\partial^{2} f_{k}}{\partial x_{i} \partial x_{j}}\left(E_{1}, m_{1}^{*}\right), b=\sum_{k, i=1}^{3} v_{k} w_{i} \frac{\partial^{2} f_{k}}{\partial x_{i} \partial m_{1}}\left(E_{1}, m_{1}^{*}\right)
$$


may be explicitly computed. Taking into account system (3.1), it follows that

$$
\begin{aligned}
& a=\frac{2 \mu}{\left(\mu+D_{1}+D_{2}+\frac{h}{c}\right)^{2}}\left[\frac{h\left(\mu_{1}+D_{1}\right)}{c^{2}}-\frac{a_{1}\left(\mu+D_{1}\right)}{b_{1} K+c_{1}}\left(D_{2}+\frac{h}{c}\right)\right. \\
& \left.\quad-\frac{\left(\mu+D_{1}\right)^{2}}{r K \mu \alpha_{1}\left(b_{1} K+c_{1}\right)}\left(D_{2}+\frac{h}{c}\right)^{2}-\frac{m_{2}(1-\alpha)}{a_{2}}\left(D_{2}+\frac{h}{c}\right)\right], \\
& b=\frac{\mu \alpha_{1} K}{\left(b_{1} K+c_{1}\right)\left(\mu+D_{1}+D_{2}+\frac{h}{c}\right)}>0 .
\end{aligned}
$$

\section{Appendix D}

Proof of Theorem 4.7 is as follows.

The second additive compound matrix of the Jacobian of the system (3.1) is given by

$$
J^{[2]}=\left(\begin{array}{ccc}
-a_{11}-a_{22} & a_{23} & a_{13} \\
a_{32} & -a_{11}-a_{33} & 0 \\
0 & a_{21} & -a_{22}-a_{33}
\end{array}\right)
$$

Let $|X|_{\infty}=\sup _{i}\left|X_{i}\right|$. The logarithmic norm $\mu_{\infty}\left(J^{[2]}\right)$ of $J^{[2]}$ endowed with the vector norm $|X|_{\infty}$ is the supremum of $-a_{11}-a_{22}+\left|a_{23}\right|+\left|a_{13}\right|,\left|a_{32}\right|-a_{11}-a_{33}$ and $\left|a_{21}\right|-a_{22}-a_{33}$.

Now, $-a_{11}-a_{22}+\left|a_{23}\right|+\left|a_{13}\right|<0$ if

$$
\frac{m_{1} U^{*}\left[b_{1} W^{*}+\left(\alpha_{1}+1\right)\left(b_{1} U^{*}+c_{1}\right)\right]}{\left(a_{1} W^{*}+b_{1} U^{*}+c_{1}\right)^{2}}<\mu+D_{1}+\frac{r U^{*}}{K}+\frac{m_{2} a_{2}(1-\alpha) W^{*}}{\left(a_{2}+V^{*}\right)^{2}}+\frac{m_{2}(1-\alpha) V^{*}}{a_{2}+V^{*}}=L_{1} .
$$

Also, $-a_{11}-a_{33}+\left|a_{32}\right|<0$ if

$$
\mu+\frac{m_{1} b_{1} U^{*} W^{*}}{\left(a_{1} W^{*}+b_{1} U^{*}+c_{1}\right)^{2}}<D_{2}+\frac{r U^{*}}{K}+\frac{h c}{\left(c+W^{*}\right)^{2}}=L_{2} .
$$

Again, $-a_{22}-a_{33}+\left|a_{21}\right|<0$ if

$$
\frac{\alpha_{1} m_{1} W^{*}\left(a_{1} W^{*}+c_{1}\right)}{\left(a_{1} W^{*}+b_{1} U^{*}+c_{1}\right)^{2}}<\mu+D_{1}+D_{2}+\frac{h c}{\left(c+W^{*}\right)^{2}}+\frac{m_{2} a_{2}(1-\alpha) W^{*}}{\left(a_{2}+V^{*}\right)^{2}}=L_{3} .
$$

Thus, if $\mu+\frac{m_{1} U^{*}\left[2 b_{1} W^{*}+\left(\alpha_{1}+1\right)\left(b_{1} U^{*}+c_{1}\right)\right]+\alpha_{1} m_{1} W^{*}\left(a_{1} W^{*}+c_{1}\right)}{\left(a_{1} W^{*}+b_{1} U^{*}+c_{1}\right)^{2}}<L$, then $\mu_{\infty}\left(J_{E^{*}}^{[2]}\right)<0$, where $L=\min \left\{L_{1}, L_{2}, L_{3}\right\}$.

Following Li and Muldowney ${ }^{65}$, the system (3.1) has no periodic solutions around $E^{*}$ provided condition (4.3) is satisfied.

Proof of Theorem 4.8 is as follows.

Consider the following positive definite function:

$$
F=\left(U-U^{*}-U^{*} \ln \frac{U}{U^{*}}\right)+\frac{p_{1}}{2}\left(V-V^{*}\right)^{2}+\frac{p_{2}}{2}\left(W-W^{*}\right)^{2},
$$


where $p_{1}$ and $p_{2}$ are positive constants, which will be chosen appropriately below. The function ' $F$ ' represents a Lyapunov function candidate corresponding to the equilibrium $E^{*}$ of the system (3.1).

Differentiating above equation with respect to time ' $t$ ' along the solution trajectories of the system (3.1), we get

$$
\begin{aligned}
\frac{d F}{d t}= & -\left[\frac{r}{K}-\frac{m_{1} b_{1} W}{\left(a_{1} W+b_{1} U+c_{1}\right)\left(a_{1} W^{*}+b_{1} U^{*}+c_{1}\right)}\right]\left(U-U^{*}\right)^{2} \\
& -p_{1}\left[\mu+D_{1}+\frac{(1-\alpha) m_{2} a_{2} W}{\left(a_{2}+V\right)\left(a_{2}+V^{*}\right)}\right]\left(V-V^{*}\right)^{2}-p_{2}\left[D_{2}+\frac{h c}{(c+W)\left(c+W^{*}\right)}\right]\left(W-W^{*}\right)^{2} \\
& +p_{1}\left[\frac{\alpha_{1} m_{1} W\left(a_{1} W^{*}+c_{1}\right)}{\left(a_{1} W+b_{1} U+c_{1}\right)\left(a_{1} W^{*}+b_{1} U^{*}+c_{1}\right)}\right]\left(U-U^{*}\right)\left(V-V^{*}\right) \\
& -\left[\frac{m_{1}\left(b_{1} U+c_{1}\right)}{\left(a_{1} W+b_{1} U+c_{1}\right)\left(a_{1} W^{*}+b_{1} U^{*}+c_{1}\right)}\right]\left(U-U^{*}\right)\left(W-W^{*}\right) \\
& +\left[p_{1}\left\{\frac{\alpha_{1} m_{1} U^{*}\left(b_{1} U+c_{1}\right)}{\left(a_{1} W+b_{1} U+c_{1}\right)\left(a_{1} W^{*}+b_{1} U^{*}+c_{1}\right)}-\frac{m_{2}(1-\alpha) V^{*}}{a_{2}+V^{*}}\right\}+p_{2} \mu\right]\left(V-V^{*}\right)\left(W-W^{*}\right) .
\end{aligned}
$$

Choose $p_{1}=p_{2}=1$, then $\frac{d F}{d t}$ can be made negative definite inside the region $\Omega$ under the conditions stated in Theorem 4.8. In this way, we have found the appropriate values for the constants in the Lyapunov function. Therefore, we conclude that the coexistence equilibrium $E^{*}$ is globally asymptotically stable.

\section{Appendix E}

Let at a critical value of $m_{1}$, say $m_{1}^{c}, A_{1}\left(m_{1}^{c}\right) A_{2}\left(m_{1}^{c}\right)-A_{3}\left(m_{1}^{c}\right)=0$. Thus, at $m_{1}=m_{1}^{c}$, the characteristic Eq. (6.3) can be written as

$$
\left(\lambda+A_{1}\right)\left(\lambda^{2}+A_{2}\right)=0 .
$$

This equation has three roots $\lambda_{1,2}= \pm i \sqrt{A_{2}}$ and $\lambda_{3}=-A_{1}$.

Thus, at $m_{1}=m_{1}^{c}$, the characteristic Eq. (6.3) has a pair of purely imaginary roots while the third root is negative. To show the transversality condition, let at any point $m_{1}$ of $\epsilon-$ neighborhood of $m_{1}^{c}, \lambda_{1,2}=\kappa\left(m_{1}\right) \pm i \rho\left(m_{1}\right)$. Substituting this in Eq. (6.3) and separating real and imaginary parts, we have

$$
\begin{aligned}
& \kappa^{3}-3 \kappa \rho^{2}+A_{1}\left(\kappa^{2}-\rho^{2}\right)+A_{2} \kappa+A_{3}=0, \\
& 3 \kappa^{2} \rho-\rho^{3}+2 A_{1} \kappa \rho+A_{2} \rho=0 .
\end{aligned}
$$

As $\rho\left(m_{1}\right) \neq 0$ from Eq. (6.6), we have

$$
\rho^{2}=3 \kappa^{2}+2 A_{1} \kappa+A_{2} .
$$

Substituting this in Eq. (6.5), we have

$$
8 \kappa^{3}+8 A_{1} \kappa^{2}+2 \kappa\left(A_{1}^{2}+A_{2}\right)+A_{1} A_{2}-A_{3}=0 .
$$

From the above equation, we get

$$
\left[\frac{d \kappa}{d m_{1}}\right]_{m_{1}=m_{1}^{c}}=-\left[\frac{1}{2\left(A_{1}^{2}+A_{2}\right)} \frac{d}{d m_{1}}\left(A_{1} A_{2}-A_{3}\right)\right]_{m_{1}=m_{1}^{c}} \neq 0 \text { provided }\left[\frac{d}{d m_{1}}\left(A_{1} A_{2}-A_{3}\right)\right]_{m_{1}=m_{1}^{c}} \neq 0
$$


To better understand the nature of the instability, we determine the initial period and the amplitude of the oscillatory solutions. Set $A_{3}=\psi A_{1} A_{2}$ in equation (6.3). Assuming that $\lambda$ depends continuously on $\psi$, we rewrite equation (6.3) as

$$
\lambda^{3}+A_{1} \lambda^{2}+A_{2} \lambda+\psi A_{1} A_{2}=0 .
$$

At $A_{3}=A_{1} A_{2}, \psi=\psi^{*}=1$, equation (6.8) factors to $\left(\lambda+A_{1}\right)\left(\lambda^{2}+A_{2}\right)$, which has solutions $\lambda\left(\psi^{*}\right)=-A_{1}$ or $\lambda\left(\psi^{*}\right)= \pm i \sqrt{A_{2}}$. This confirms the existence of Hopf-bifurcation.

Observe that in this new setting, $0 \leq \psi \leq \psi^{*}$ is equivalent to $0 \leq m_{1} \leq m_{1}^{c}$, $0 \leq \psi=\psi^{*}$ is equivalent to $m_{1}=m_{1}^{c}$, and $\psi \geq \psi^{*}$ is equivalent to $m_{1} \geq m_{1}^{c}$. If we set $\psi=\psi^{*}+\epsilon^{2} \xi$, where $|\epsilon| \ll 1$ and $\xi= \pm 1$, then $\lambda(\psi)=\lambda\left(\psi^{*}+\epsilon^{2} \xi\right)$ so that the linear portion in $\epsilon^{2} \xi$ of the Taylor series expansion of $\lambda$ about $\psi^{*}$ is

$$
\lambda(\psi)=\lambda\left(\psi^{*}\right)+\lambda^{\prime}\left(\psi^{*}\right) \epsilon^{2} \xi+O\left(\epsilon^{4}\right),
$$

where prime denotes differentiation with respect to $\psi$. Differentiating and simplifying equation (6.9) yields

$$
\lambda^{\prime}(\psi) \equiv \frac{A_{1} A_{2}}{2\left(A_{1}^{2}+A_{2}\right)} \pm i \frac{A_{1}^{2} \sqrt{A_{2}}}{2\left(A_{1}^{2}+A_{2}\right)} .
$$

Using the fact that $\Re\left(\lambda\left(\psi^{*}\right)\right)=0$ and $\Re\left(\lambda^{\prime}\left(\psi^{*}\right)\right)=\frac{A_{1} A_{2}}{2\left(A_{1}^{2}+A_{2}\right)}>0$, and substituting $\lambda\left(\psi^{*}\right)$ and $\lambda^{\prime}(\psi)$ in equation (6.9), we obtain the approximation

$$
\begin{aligned}
\lambda(\psi) & =\lambda\left(\psi^{*}\right)+\lambda^{\prime}\left(\psi^{*}\right) \epsilon^{2} \xi \\
& =\frac{A_{1} A_{2} \epsilon^{2} \xi}{2\left(A_{1}^{2}+A_{2}\right)} \pm i \sqrt{A_{2}}\left(1+\frac{A_{1}^{2} \epsilon^{2} \xi}{2\left(A_{1}^{2}+A_{2}\right)}\right)+O\left(\epsilon^{4}\right) .
\end{aligned}
$$

Thus, the initial period and amplitude of the oscillations associated with the loss of stability when $\psi>\psi^{*}$ are $\frac{2 \pi}{\sqrt{A_{2}}\left(1+\frac{A_{1}^{2} \epsilon^{2} \xi}{2\left(A_{1}^{2}+A_{2}\right)}\right)}$ and $\exp \left(\frac{A_{1} A_{2} \epsilon^{2} \xi}{2\left(A_{1}^{2}+A_{2}\right)}\right)$, respectively where $\epsilon=\sqrt{\frac{\left|\psi-\psi^{*}\right|}{|\xi|}}$.

We investigate the orbital stability of Hopf-bifurcating periodic solution by using Poore's sufficient condition ${ }^{66}$. The supercritical and subcritical nature of Hopfbifurcating periodic solution is determined by the positive/negative sign of the real part of $\Phi$, where

$\Phi=-a_{l} G_{u_{j} u_{m} u_{s}}^{l} b_{j} b_{m} \bar{b}_{s}+2 a_{l} G_{u_{j} u_{m}}^{l} b_{j}\left(J_{E^{*}}^{-1}\right)_{m r} G_{u_{p} u_{q}}^{r} b_{q} \bar{b}_{q}+a_{l} G_{u_{j} u_{k}}^{l} \bar{b}_{j}\left[\left(J_{E^{*}}-2 i \omega_{0}\right)^{-1}\right]_{k r} G_{u_{p} u_{q}}^{r} b_{p} b_{q}$.

Here, the repeated indices within each term imply a sum from 1 to 3 and all the derivatives of $G^{l}$ are evaluated at the equilibrium $E^{*}$ with $u_{1}=U, u_{2}=V, u_{3}=$ $W$, and $J_{E^{*}}$ is the Jacobian matrix of system $(3.1)$ calculated at $E^{*}$. $\left[\left(J_{E^{*}}\right)^{-1}\right]_{m r}$ denotes the element in row $m$ and column $r$ of $\left(J_{E^{*}}\right)^{-1}$. Here, $a=\left(a_{1}, a_{2}, a_{3}\right)$ and $b=\left(b_{1}, b_{2}, b_{3}\right)^{T}$ are left and right normalized eigenvectors of $J_{E^{*}}$ with respect to the eigenvalues $\pm i \omega_{0}$ at $m_{1}=m_{1}^{c}$ so that $a . b=1$. 
The left and right normalized eigenvectors are given by

$$
a=\frac{\xi_{1}}{\mu G_{U}^{2}}\left(\mu G_{U}^{2}, \mu\left(i \omega_{0}-G_{U}^{1}\right),\left(G_{U}^{1} G_{V}^{2}-\omega_{0}^{2}\right)-i \omega_{0}\left(G_{U}^{1}+G_{V}^{2}\right)\right)
$$

and

$$
b=\frac{\xi_{2}}{\mu G_{W}^{1}}\left(\mu G_{W}^{1},\left(G_{U}^{1} G_{W}^{3}-\omega_{0}^{2}\right)-i \omega_{0}\left(G_{U}^{1}+G_{W}^{3}\right), \mu\left(i \omega_{0}-G_{U}^{1}\right)\right)^{T}
$$

where $\xi_{1}$ and $\xi_{2}$ are complex numbers.

Using $a . b=1$, we obtain $\xi_{1} \xi_{2}$. If $[\Phi]_{m_{1}=m_{1}^{c}}>0$, the system (3.1) undergoes a supercritical Hopf-bifurcation as $m_{1}$ increases through $m_{1}^{c}$, so that the bifurcating periodic orbit is asymptotically orbitally stable.

\section{Appendix F}

Jacobian of the system $(2.1)$ evaluated at $E^{*}$ leads to the following characteristic equation:

$$
\operatorname{det}\left(J_{0}+e^{-\lambda \tau} J_{\tau}-\lambda I_{3}\right)=0
$$

where $I_{3}$ is an identity matrix of order 3 and

$$
J_{0}=\left(\begin{array}{ccc}
V_{1} & 0 & V_{2} \\
0 & V_{3} & V_{4} \\
0 & V_{5} & V_{6}
\end{array}\right), J_{\tau}=\left(\begin{array}{ccc}
0 & 0 & 0 \\
M_{1} & 0 & M_{2} \\
0 & 0 & 0
\end{array}\right)
$$

with

$$
\begin{aligned}
& V_{1}=-\left(\frac{r U^{*}}{K}-\frac{m_{1} b_{1} U^{*} W^{*}}{\left(a_{1} W^{*}+b_{1} U^{*}+c_{1}\right)^{2}}\right), V_{2}=-\frac{m_{1} U^{*}\left(b_{1} U^{*}+c_{1}\right)}{\left(a_{1} W^{*}+b_{1} U^{*}+c_{1}\right)^{2}}, \\
& V_{3}=-\left(\mu+D_{1}+\frac{m_{2} a_{2}(1-\alpha) W^{*}}{\left(a_{2}+V^{*}\right)^{2}}\right), V_{4}=-\frac{m_{2}(1-\alpha) V^{*}}{a_{2}+V^{*}}, V_{5}=\mu, \\
& V_{6}=-\left(D_{2}+\frac{h c}{\left(c+W^{*}\right)^{2}}\right), M_{1}=\frac{\alpha_{1} m_{1} W^{*}\left(a_{1} W^{*}+c_{1}\right)}{\left(a_{1} W^{*}+b_{1} U^{*}+c_{1}\right)^{2}}, M_{2}=\frac{\alpha_{1} m_{1} U^{*}\left(b_{1} U^{*}+c_{1}\right)}{\left(a_{1} W^{*}+b_{1} U^{*}+c_{1}\right)^{2}} .
\end{aligned}
$$

Now the Jacobian matrix of the delay model (2.1) around the equilibrium point $E^{*}$ is

$$
J_{E^{*}}=\left(\begin{array}{ccc}
V_{1}-\lambda & 0 & V_{2} \\
M_{1} e^{-\lambda \tau} & V_{3}-\lambda & V_{4}+M_{2} e^{-\lambda \tau} \\
0 & V_{5} & V_{6}-\lambda
\end{array}\right)
$$

The characteristic equation of the delay system at the equilibrium point $E^{*}$ is

$$
\lambda^{3}+C_{1} \lambda^{2}+C_{2} \lambda+C_{3}=\left[D_{1} \lambda+D_{2}\right] e^{-\lambda \tau},
$$

where

$$
\begin{aligned}
& C_{1}=-\left(V_{1}+V_{2}+V_{3}\right), C_{2}=V_{1}\left(V_{3}+V_{6}\right)+V_{3} V_{6}-V_{4} V_{5}, C_{3}=V_{1}\left(V_{4} V_{5}-V_{3} V_{6}\right), \\
& D_{1}=V_{5} M_{2}, D_{2}=V_{5}\left(V_{2} M_{1}-V_{1} M_{2}\right) .
\end{aligned}
$$


The coexistence equilibrium point $E^{*}$ will be locally asymptotically stable if all roots of the corresponding characteristic equation (6.12) are negative or having negative real parts. The classical Routh-Hurwitz criterion cannot be used to investigate the stability of the system (2.1) as Eq. (6.12) is a transcendental equation, and it has infinitely many roots. To determine the nature of the stability, we require the signs of the real parts of the roots of the characteristic equation (6.12). Let $\lambda(\tau)=$ $\beta(\tau)+i \omega(\tau)$ be eigenvalue of the characteristic equation (6.12). Substituting this value in Eq. (6.12), we obtain real and imaginary parts, respectively as

$$
\begin{aligned}
\beta^{3}-3 \beta \omega^{2}+C_{1}\left(\beta^{2}-\omega^{2}\right)+C_{2} \beta+C_{3} & =\left[\left(D_{1} \beta+D_{2}\right) \cos (\omega \tau)+D_{1} \omega \sin (\omega \tau)\right] e^{-\beta \tau}, \\
-\omega^{3}+3 \beta^{2} \omega+2 C_{1} \beta \omega+C_{2} \omega & =\left[D_{1} \omega \cos (\omega \tau)-\left(D_{1} \beta+D_{2}\right) \sin (\omega \tau)\right] e^{-\beta \tau} .
\end{aligned}
$$

A necessary condition for a stability change of $E^{*}$ is that the characteristic equation (6.12) should have purely imaginary roots. Putting $\beta=0$ in Eqs. (6.13) and (6.14), we have

$$
\begin{aligned}
-C_{1} \omega^{2}+C_{3} & =D_{2} \cos (\omega \tau)+D_{1} \omega \sin (\omega \tau), \\
-\omega^{3}+C_{2} \omega & =D_{1} \omega \cos (\omega \tau)-D_{2} \sin (\omega \tau) .
\end{aligned}
$$

Eliminating $\tau$ by squaring and adding Eqs. (6.15) and (6.16), we get the algebraic equation for determining $\omega$ as

$$
\omega^{6}+\left(C_{1}^{2}-2 C_{2}\right) \omega^{4}+\left(C_{2}^{2}-2 C_{1} C_{3}-D_{1}^{2}\right) \omega^{2}+\left(C_{3}^{2}-D_{2}^{2}\right)=0 .
$$

Substituting $\omega^{2}=\theta$ in Eq. (6.17), we obtain the following cubic equation:

$$
\kappa(\theta)=\theta^{3}+\sigma_{1} \theta^{2}+\sigma_{2} \theta+\sigma_{3}=0,
$$

where

$$
\sigma_{1}=C_{1}^{2}-2 C_{2}, \sigma_{2}=C_{2}^{2}-2 C_{1} C_{3}-D_{1}^{2}, \sigma_{3}=C_{3}^{2}-D_{2}^{2}
$$

Now, if $\sigma_{1}$ and $\sigma_{3}$ are of opposite signs then by Descarte's rule of sign, Eq. (6.18) has at least one positive root for either sign of $\sigma_{2}$.

Since $\omega_{0}$ is a solution of Eq. (6.17), the characteristic equation (6.12) has pair of purely imaginary roots $\pm i \omega_{0}$. From Eqs. (6.15) and (6.16), we have $\tau_{p}^{*}$ is a function of $\omega_{0}$ for $p=0,1,2, \cdots$, which is given by

$$
\tau_{p}^{*}=\frac{1}{\omega_{0}} \arccos \left[\frac{D_{1} \omega_{0}\left(C_{2} \omega_{0}-\omega_{0}^{3}\right)+D_{2}\left(C_{3}-C_{1} \omega_{0}^{2}\right)}{D_{1}^{2} \omega_{0}^{2}+D_{2}^{2}}\right]+\frac{2 \pi p}{\omega_{0}} .
$$

Now, the system will be locally asymptotically stable around the coexistence equilibrium $E^{*}$ for $\tau=0$ if $\sigma_{1} \sigma_{2}-\sigma_{3}>0$. In that case by Butler's lemma, the equilibrium $E^{*}$ will remain stable for $\tau<\tau^{*}$, such that $\tau^{*}=\min _{p \geq 0} \tau_{p}^{*}$ and the equilibrium $E^{*}$ will be unstable for $\tau \geq \tau^{*}$, provided the transversality condition holds. 
Now, we verify the transversality condition

$$
\left[\frac{d R e(\lambda)}{d \tau}\right]_{\tau=\tau^{*}}>0 .
$$

Differentiating Eqs. (6.13) and (6.14) with respect to $\tau$ and putting $\beta=0$, we obtain

$$
\begin{aligned}
R(\omega) \frac{d \beta}{d \tau}+S(\omega) \frac{d \omega}{d \tau} & =U(\omega), \\
-S(\omega) \frac{d \beta}{d \tau}+R(\omega) \frac{d \omega}{d \tau} & =W(\omega),
\end{aligned}
$$

where

$$
\begin{aligned}
& R(\omega)=-3 \omega^{2}+C_{2}-D_{1} \cos (\omega \tau)+\tau\left[D_{2} \cos (\omega \tau)+D_{1} \omega \sin (\omega \tau)\right], \\
& S(\omega)=-2 C_{1} \omega+D_{2} \tau \sin (\omega \tau)-D_{1} \sin (\omega \tau)-D_{1} \tau \omega \cos (\omega \tau), \\
& U(\omega)=-D_{2} \omega \sin (\omega \tau)+D_{1} \omega^{2} \cos (\omega \tau), \\
& W(\omega)=-D_{1} \omega^{2} \sin (\omega \tau)-D_{2} \omega \cos (\omega \tau) .
\end{aligned}
$$

Solving the above Eqs., we have

$$
\left[\frac{d R e(\lambda)}{d \tau}\right]_{\tau=\tau^{*}}=\left[\frac{U(\omega) R(\omega)-S(\omega) W(\omega)}{R^{2}(\omega)+S^{2}(\omega)}\right]_{\tau=\tau^{*}},
$$

which shows that $\left[\frac{d R e(\lambda)}{d \tau}\right]_{\tau=\tau^{*}}>0$ if $U(\omega) R(\omega)-S(\omega) W(\omega)>0$. Thus, Hopf bifurcation occurs at $\tau=\tau^{* 30}$. 